\title{
A Game-theoretic Framework for Network Coding Based Device-to-Device Communications
}

\author{
Ahmed Douik, Student Member, IEEE, Sameh Sorour, Senior Member, IEEE, Hamidou Tembine \\ , Senior Member, IEEE, Tareq Y. Al-Naffouri, Member, IEEE, and Mohamed-Slim Alouini, Fellow, IEEE
}

\begin{abstract}
This paper investigates the delay minimization problem for instantly decodable network coding (IDNC) based deviceto-device (D2D) communications. In D2D enabled systems, users cooperate to recover all their missing packets. The paper proposes a game theoretic framework as a tool for improving the distributed solution by overcoming the need for a central controller or additional signaling in the system. The session is modeled by self-interested players in a non-cooperative potential game. The utility functions are designed so as increasing individual payoff results in a collective behavior achieving both a desirable system performance in a shared network environment and the Nash equilibrium. Three games are developed whose first reduces the completion time, the second the maximum decoding delay and the third the sum decoding delay. The paper, further, improves the formulations by including a punishment policy upon collision occurrence so as to achieve the Nash bargaining solution. Learning algorithms are proposed for systems with complete and incomplete information, and for the imperfect feedback scenario. Numerical results suggest that the proposed game-theoretical formulation provides appreciable performance gain against the conventional point-to-multipoint (PMP), especially for reliable user-to-user channels.
\end{abstract}

Index Terms-Device-to-device communications, instantly decodable network coding, non-cooperative games, potential game, Nash equilibrium.

\section{INTRODUCTION}

\section{A. Network Coding}

$\mathrm{N}^{2}$ ETWORK Coding (NC) [2] is a promising technique to improve the throughput of communication systems significantly. The benefits are even more pronounced in wireless erasure networks due to the broadcast nature of the transmissions. Such merits are essential for real-time applications requiring reliable communications and fast recovery over erasure channels, such as multimedia streaming [3].

Network coding schemes can mainly be divided into two groups, i.e., the Random Network Coding (RNC), and the

A part of this paper [1] is published in proc. of IEEE Global Telecommunications Conference (GLOBECOM' 2014), Austin, Texas, USA.

Ahmed Douik is with the Department of Electrical Engineering, California Institute of Technology, Pasadena, CA 91125 USA (e-mail: ahmed.douik@caltech.edu).

Sameh Sorour is with the Department of Electrical and Computer Engineering, University of Idaho, Moscow, ID 83844, USA (e-mail: samehsorour@gmail.com).

Hamidou Tembine is with the Electrical and Computer Engineering Department at New York University Abu Dhabi (NYUAD), Abu Dhabi 129188, United Arab Emirates (email: tembine@ieee.org).

T. Y. Al-Naffouri is with King Abdullah University of Science and Technology, Thuwal 23955-6900, Saudi Arabia, and also with King Fahd University of Petroleum and Minerals, Dhahran 31261, Saudi Arabia (e-mail: tareq.alnaffouri@kaust.edu.sa).

M.-S. Alouini is with the Division of Computer, Electrical and Mathematical Sciences and Engineering, King Abdullah University of Science and Technology, Thuwal 23955-6900, Saudi Arabia (e-mail: slim.alouini@kaust.edu.sa).
Opportunistic Network Coding (ONC). RNC combines packets using independent, random, and non-zero coefficients [4] that results in an optimality in the number of transmissions for broadcast applications and an ability to recover even without feedback [5]. However, RNC is not suitable for realtime applications as not only it does not allow progressive decoding but it also requires expensive computations at the receiver to decode the frame. On the other hand, ONC mixes packets according to their received/lost state at each user [6]. Such technique is shown to be a graceful solution for packet recovery for wireless networks [7].

Instantly Decodable Network Coding (IDNC), an ONC subclass, suits most of the real-time applications thanks to its instantaneous and progressive decoding of packets. IDNC is implemented using binary XOR to both encode and decode packets. Furthermore, no buffer is needed at receivers as noninstantly decodable packets are not stored for future decoding possibilities. In the last few years, IDNC is an intensive subject of research [8]-[21]. While the authors in [8] investigate the sum decoding delay reduction for perfect feedback, the authors in [9], [17], [18], [22] study the effects of feedback imperfection on the sum decoding delay minimization. The maximum decoding delay is introduced in [19] as a more reliable delay metric in IDNC. The completion time is reduced in [21] and is shown to be related to the maximum decoding delay in [20] and thus, be better controlled through it.

\section{B. Motivation and Related Work}

In all aforementioned works, the base station of a Point-toMultipoint (PMP) network, e.g., cellular, WiFi, WiMAX and roadside to vehicle systems, is responsible for the recovery of erased packets. This can pose a threat to the resources of such base stations and their abilities to deliver the required enormous data rates, especially in the next generation mobile radio system $(5 \mathrm{G})$ [23]. The problem becomes more severe in roadside to vehicle networks as the vehicles usually bypass the roadside senders very fast and thus cannot rely on it for packet recovery. An elegant alternative to the problem is the notion of device-to-device (D2D) communications introduced in [24]. In such configuration, users cooperate by sending coded packets to each other over short-range and potentially more reliable communication channels. Furthermore, D2D systems enable fast and reliable data communications over ad-hoc networks, such as vehicular and sensor networks. Consequently, delays minimization in such IDNC-based D2D systems is crucial.

Consider a set of geographically close users collaborating to complete the recovery of their missing packets. Unlike PMP systems that require only an optimization over the packet combination, IDNC-based D2D networks need an extra 
optimization over the transmissing user due to the asymmetry of both the received packets and the D2D channels. Recently Aboutorab et al. [12] consider the problem of minimizing the sum decoding delay for an IDNC-based D2D system in a centralized fashion. The term centralized refers to a configuration in which a central unit, e.g., the base station in a cellular network, decides on the transmitting user and the packet combination. Despite being efficient, such centralized optimization requires a central controller which may not be always feasible, e.g., roadside to vehicle systems. Further, a substantial amount of signaling is required to coordinate such centralized system. This paper proposes to overcome the mentioned above limitations by introducing an entirely distributed system and modeling the system by a cooperative control problem.

Cooperative control problems entail numerous autonomous users seeking to achieve a global objective collectively. The network coding problem is one example of cooperative control problems, in which the global goal is for all users to use a shared resource efficiently by opportunistically taking advantage of the possible coding occasions. The central challenge in cooperative control problems is to derive a local control mechanism for the individual users such that each operates in a manner that collectively serves the desired global objective.

\section{Contributions}

This paper's main contribution is to propose a fully distributed solution to the delays minimization in IDNC-based D2D networks. The paper investigates three important metrics in IDNC namely the completion time, the maximum decoding delay and the sum decoding delay. A game theoretic framework is introduced to studying such delays wherein game theory is used as a tool for improving the distributed solution by overcoming the need for a central controller or additional signaling in the system. To the best of our knowledge, using game theory tools to model IDNC-based D2D communications is not addressed in the literature, and only heuristic algorithms are proposed to solve the problem in a centralized fashion [12]. Moreover, this work serves as a background to build more complicated system such as the multicast scenario, i.e., the packet demand of each user can differ, or the partially connected scenario, i.e., users are not all in the transmission range of each other.

The paper solves the problems by deriving expressions for the individual utility functions of each delay metric in such a way that increasing personal payoff results in a collective behavior achieving a desirable system performance in a shared network environment. Even though such formulation reaches the Nash equilibrium, it is not stable as it deviates upon packet collisions. Therefore, a punishment policy is included so as to improve the games formulations and restrain the set of equilibrium to the one-dimensional line containing the Nash bargaining solution of interest. The paper, further, proposes learning algorithms to operate at one of the equilibrium points of systems with either complete or incomplete information.

The aforementioned works consider a perfect and prompt feedback from all the users. Such assumption is too idealistic given the impairments on the feedback link [25] of wireless networks, due to shadowing, high interference, and fading. In that situation, users need to transmit several subsequent packets having only partial information about their reception status at the other users. Moreover, the scenario introduces a new dimension to the problem since the information at the different users is no longer common knowledge. In such uncertainties and non-symmetric information, users are no longer sure about the outcome of their actions, and thus are not confident about the resulting completion time and decoding delays. The paper proves that the proposed framework can be easily extensible to imperfect feedback scenarios and provides as an example a learning algorithm for the lossy feedback case.

The rest of this paper is divided as follows: The system parameters and problem formulation are provided in Section II. Section III briefly recalls background about potential games, designs the utility functions, and investigates the game equilibria. The punishment policy and the new game formulations are provided in Section IV. In Section V, the learning algorithms are illustrated for systems with perfect and imperfect information and feedback. Before concluding in Section VII, simulations results are discussed in Section VI.

\section{Notations}

The following notations are adopted in this paper. The notation $\mathbf{X}$ refers to a matrix whose $i$-th column is $\underline{X}_{i}$. The notation $\underline{x}$ denotes to a vector whose $i$-th entry is $x_{i}$. The superscript ${ }^{\mathbf{T}}$ represents the transpose operator. Let $\{0,1\}^{x \times y}$ denotes the set of binary matrices of dimension $x \times y$ and let $\{0,1\}^{x}$ denotes the set of binary vectors of length $x$. The notation $\left[\underline{X}_{1}, \ldots, \underline{X}_{n}\right]$ refers to the matrix whose $i$-th column is the vector $\underline{X}_{i}$ and the notation $\mathbf{X}=\left[x_{i j}\right]$ refers to a matrix $\mathbf{X}$ whose $i$-th row and $j$-th column is the element $x_{i j}$. Finally, a function $f$ that maps elements $x \in X$ to the element $f(x) \in Y$ may be written as follows:

$$
f: \mid \begin{array}{ccc}
X & \longrightarrow & Y \\
x & \longmapsto & f(x) .
\end{array}
$$

\section{System Model and Problems Formulation}

This section first presents the considered system model, parameters, and protocol. Afterward, the definitions and expressions of the IDNC metrics investigated in this paper, i.e., the completion time and the decoding delay, are provided. Finally, the section formulates the delays minimization problems.

\section{A. System Model}

Consider a wireless network composed of a set $\mathcal{M}=$ $\{1, \ldots, M\}$ of $M$ geographically close users interested in receiving a frame $\mathcal{N}=\{1, \ldots, N\}$ of $N$ source packets that the base-station (BS) holds. The terms user and player are used interchangeably throughout this paper. In a D2D-enabled network, players cooperate to obtain all the packets of the frame regardless of the order. This document assumes singlehop transmissions, which means that all the actors are in the transmission range of each other.

The physical layers of both the sender and the receivers are abstracted by memory-less erasure channels. Let $q_{i}$ denote the 
packet erasure probability, i.e., the probability to lose a packet, player $i$ is experiencing during a transmission from the BS and let $\mathbf{P}=\left[p_{i j}\right], i, j \in \mathcal{M}$ denote the erasure from player $j$ to player $i$. All the packet erasure probabilities are assumed to be constant during the transmission of the frame. Further, these probabilities are assumed to be known to both the BS and the players.

In single-hop transmissions, simultaneous communications are prohibited as they result in interference at all receivers. Therefore, at each time slot, the packet combination, and the transmitting player are chosen according to the diversity of lost/received packets and the expected erasure patterns of the links. Players that successfully received and decoded a packet send an acknowledgment (ACK) with the appropriate frequency and modulation so that its reception is perfect. Since all actors can already overhear all the feedback sent by the other players, then the system does not require any additional feedback load.

\section{B. Initialization and Recovery Phases}

At the start of the session, all players are requesting the $N$ packets held by the BS. In an initialization phase, i.e., the first $N$ time slots, the BS broadcasts the $N$ source packets uncoded. At the end of this period, each packet of the frame is at least acknowledged by one of the players. Otherwise, such packet is re-transmitted by the BS until the condition is reached. For each player $i$, the packets of the frame $\mathcal{N}$ can be in one of the following sets:

- The Has set (denoted by $\mathcal{H}_{i}$ ): The sets of packets successfully received by player $i$.

- The Wants set (denoted by $\mathcal{W}_{i}=\mathcal{N} \backslash \mathcal{H}_{i}$ ): The sets of packets erased at player $i$.

These informations are stored in a state matrix (SM) $\mathbf{S}=\left[s_{i j}\right]$ such that $s_{i j}=0$ if packet $j$ is successfully received by user $i$ (i.e., $j \in \mathcal{H}_{i}$ ) and 1 otherwise. After the initial transmission, the recovery phase starts. In this phase, the players cooperate to recover their missing packets by transmitting to each other binary XOR encoded packets of the source packets they already received.

In the recovery phase, a packet combination $\underline{\kappa}$ is innovative for a player $i$ if he can decode the combination and retrieve a new source packet from it. Given that encoding is performed using the binary field $\mathbb{F}_{2}$, it can be easily seen that a packet combination is innovative for player $i$ if and only if it contains a single packet from $\mathcal{W}_{i}$. Therefore, the set of players $\underline{\tau}(\underline{\kappa})=$ $\left(\tau_{1}, \cdots, \tau_{M}\right)$ to whom the packet mix $\underline{\kappa}$ is innovative, called the targeted players, is defined as follows:

$$
\tau_{i}(\underline{\kappa})= \begin{cases}1 & \text { if } \sum_{j=1}^{N} s_{i j} \kappa_{j}=1 \\ 0 & \text { otherwise, }\end{cases}
$$

where $\kappa_{j}=1$ if packet $j$ is included in the combination $\kappa$ and 0 otherwise.

After each transmission, targeted players send acknowledgments consisting of one bit indicating the successful reception. This process is repeated until all players report that they obtained all the packets, i.e., until $\mathbf{S}=\mathbf{0}$.

\section{Decoding Delay and Completion Time Expressions}

Due to the well-known throughput-delay tension in NC [26], [27], a higher information flow in the network, i.e., throughput, does not always translate into lower delay as packets need to be extracted before decoding. Balancing these two aspects being challenging [28], this paper proposes optimizing each separately. While throughput is measured, in IDNC, using the completion time for their inverse proportionality under fixed packet size, delay, in IDNC, is measured through the decoding delay. The definitions of these metrics are provided in what follows:

Definition 1. The individual completion time $\mathcal{C}_{i}$ of player $i$ is the number of recovery transmissions required until he obtains its requested packets. The overall completion time $\mathcal{C}=\max _{i \in \mathcal{M}} \mathcal{C}_{i}$ is the number of recovery transmissions required until all users obtain their requested packets.

Definition 2. At any transmission, a player $i$, with non-empty Wants set, experiences a one unit increase of decoding delay if not exactly one player transmitted or if he receives a noninnovative packet.

Let $M$ be a binary vector of size $M$ whose $i$-th entry is 0 if player $i$ have an empty Wants set, i.e., $\mathcal{W}_{i}=\varnothing$ and 1 otherwise, and let $\bar{\tau}(\underline{\kappa})=\underline{1}-\underline{\tau}(\underline{\kappa})$ be the set of non-targeted players by the packet mix $\kappa$. The state $\boldsymbol{\omega} \in \Omega$ of the system, i.e., the different erasure patterns of the links between each couple of players, can be described by the following formula:

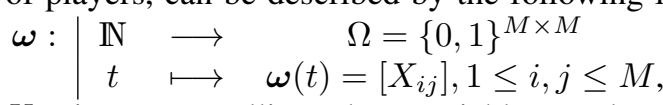

where $X_{i j}$ is a Bernoulli random variable equal to 0 with probability $p_{i j}$.

Let $\underline{a}_{t}$ be a binary vector of length $M$ whose $i$-th element is equal to 1 if player $i$ is transmitting and let $\underline{\mathcal{D}}\left(\underline{a}_{t}\right)$ be the decoding delay experienced in the $t$-th recovery transmission. From Definition 2, simultaneous transmissions results in an increase of the decoding delay of all users with non-empty Wants set. Therefore, for any $\underline{a}_{t} \in\{0,1\}^{M}$ such that $\left\|\underline{a}_{t}\right\|_{1} \neq$ 1 , the decoding delay is $\underline{\mathcal{D}}\left(\underline{a}_{t}\right)=\underline{M}$. Assuming only player $i$ is transmitting, player $j$ with non-empty Wants set (i.e., $\underline{M}_{j}=$ 1 ), who successfully receives (i.e., $X_{i j}=1$ ) a non-innovative packet (i.e., $\bar{\tau}_{j}=1$ ) experiences one unit of decoding delay. Therefore, for player $i$ the sole transmitting user, the vector $\underline{\mathcal{D}}\left(\underline{a}_{t}\right)$ can be expressed as follows:

$$
\underline{\mathcal{D}}\left(\underline{a}_{t}\right): \mid \begin{array}{ccc}
\{0,1\}^{M+N} & \longrightarrow & \mathbb{N}^{M} \\
\left(\underline{\omega}_{i}(t), \underline{\kappa}\right) & \longmapsto & \underline{\omega}_{i}(t) \circ \underline{\bar{\tau}}(\underline{\kappa}) \circ \underline{M},
\end{array}
$$

where $\circ$ is the Hadamard product. The cumulative decoding delays $\underline{\mathbb{D}}\left(\underline{a}_{t}\right)$ experienced by all players, since the beginning of the recovery phase until the $t$-th transmission, can be written as follows:

$$
\underline{\mathbb{D}}\left(\underline{a}_{t}\right)= \begin{cases}\underline{\mathbb{D}}(t-1)+\underline{M} & \text { if }\left\|\underline{a}_{t}\right\|_{1} \neq 1 \\ \underline{\mathbb{D}}(t-1)+\underline{\mathcal{D}}\left(\underline{a}_{t}\right) & \text { otherwise }\end{cases}
$$

The problem of minimizing the completion time for the whole recovery phase is shown to be intractable in [20]. A traditional approach is to approximate the completion time, at each transmission, by an expression involving the decoding delay as shown in the following corollary. 
Corollary 1. The completion time for player $i$ can be approximated at the t-th transmission by the following quantity:

$$
\mathcal{C}_{i}\left(\underline{a}_{t}\right)=\frac{\left|\mathcal{W}_{i}(0)\right|+\mathbb{D}_{i}\left(\underline{a}_{t}\right)-\tilde{p}_{i}}{1-\tilde{p}_{i}}
$$

where $\mathcal{W}_{i}(0)$ is the Wants set of player $i$ at the beginning of the recovery phase and $\tilde{p}_{i}$ is the average erasure probability linking the player $i$ to the other players.

Proof: The proof of this corollary is omitted as it mirrors the steps used in [20]. Whereas in [20] only the BS is transmitting, in a D2D configuration all the player can communicate and thus the probability for a packet to be erased at the $i$ th player is approximated by the average erasure probability linking him to all other players.

\section{Problems Formulation}

Let $\underline{\mathcal{C}}\left(\underline{a}_{t}\right)$ be the vector of size $M$ containing all the individual anticipated completion time defined in (6). From Definition 1, the overall completion time can be written as $\mathcal{C}\left(\underline{a}_{t}\right)=\max _{i \in \mathcal{M}} \mathcal{C}_{i}\left(\underline{a}_{t}\right)=\left\|\underline{\mathcal{C}}\left(\underline{a}_{t}\right)\right\|_{\infty}$. Therefore, the completion time reduction problem at the $t$-th transmission in IDNC-based D2D network can be expressed as follows:

$$
\min _{\underline{a}_{t} \in\{0,1\}^{M}}\left\{\min _{\kappa \in\{0,1\}^{N}}\left\|\underline{\mathcal{C}}\left(\underline{a}_{t}\right)\right\|_{\infty}\right\} .
$$

For a fixed transmitting player $i$, the expression optimal packet combination $\underline{\kappa}^{i}$ such user can generate in order to minimize the anticipated completion time (7) is given in [20]. In a similar way, the maximum and sum decoding delay reduction problem can be written, at the $t$-th transmission in IDNC-based D2D network, respectively as follows:

$$
\begin{gathered}
\left.\min _{\underline{a}_{t} \in\{0,1\}^{M}}\left\{\min _{\kappa \in\{0,1\}^{N}} \| \underline{\mathbb{D}}\left(\underline{a}_{t}\right)\right) \|_{\infty}\right\} . \\
\left.\min _{\underline{a}_{t} \in\{0,1\}^{M}}\left\{\min _{\kappa \in\{0,1\}^{N}} \| \underline{\mathbb{D}}\left(\underline{a}_{t}\right)\right) \|_{1}\right\} .
\end{gathered}
$$

The expression of the optimal packet combination that a player $i$ is the only transmitting users in order to minimize the maximum and the sum decoding delay can be found respectively in [19] and [8]. Given the expressions of the optimal packet combinations, any transmitting player is assumed to generate the optimal packet mix that reduces the delay metric under investigation.

The rest of the paper proposes formulating the above optimization problems by non-cooperative games so as to reach their optimal solution using fully distributed fashion. In the next section, the games are formulated using the objective function defined in (7), (8), and (9) and their equilibrium computed. As these games are unstable for systems with incomplete information, the paper suggests integrating a punishment policy in the game formulation so as to reach the Nash bargaining solution and increase the stability of the systems. Finally, learning algorithms are proposed to operate at one of the equilibrium points of systems with either complete or incomplete information and feedback.

\section{Games Formulation And Equilibrium Analysis}

This section formulates the delay reduction problems in IDNC-based D2D systems as potential games. Background about non-cooperative and potential games and their equilibria are presented in the first part of this section. Afterwards, the games are designed using the objective function defined in (7), (8), and (9) and their equilibrium computed.

\section{A. Background: Non-cooperative and Potential Games}

1) Definitions and Notations: A finite stochastic noncooperative game is defined [29] by the following 6-uplet:

$$
\mathcal{G}=\left(\mathcal{M},\left\{\overline{\mathcal{A}}_{i}\right\}, \Omega,\left\{\mathcal{A}_{i}\right\},\left\{\alpha_{i}\right\},\left\{\mathcal{U}_{i}\right\}\right),
$$

with:

- $\mathcal{M}=\{1, \ldots, M\}$ is the set of players,

- $\overline{\mathcal{A}}_{i}$ is the set of all possible actions for player $i$ during the course of the game,

- $\Omega$ is the set of possible states of the game,

- $\mathcal{A}_{i}(\omega)$ is the set of possible action for player $i$ in the state $w \in \Omega$ of the game,

- $\alpha_{i}: \Omega \longrightarrow 2^{\mathcal{A}_{i}}$ is the correspondence determining the possible actions at a given state of a game,

- $\mathcal{U}_{i}$ is the utility function of player $i$.

Let $\omega(t) \in \Omega$ be the state of the game at the stage $t$. For notation simplicity, the set of actions of player $i$ at stage $t$ is denoted by $\mathcal{A}_{i}(t)$ instead of $\mathcal{A}_{i}(\omega(t))$. Let $\mathcal{A}(t)=\prod_{i} \mathcal{A}_{i}(t)$ be the set of all possible actions at the stage $t$ of the game.

For an action profile $\underline{a}_{t}=\left(a_{1}(t), \ldots, a_{M}(t)\right)^{\mathbf{T}} \in \mathcal{A}(t)$, let $\underline{a}_{t,-i}$ denotes the profile of players other than player $i$, i.e., $\underline{a}_{t,-i}=\left(a_{1}(t), \ldots, a_{i-1}(t), a_{i+1}(t), \ldots a_{M}(t)\right)^{\mathbf{T}}$. A profile $\underline{a}_{t}$ of actions may be written as $\left(\underline{a}_{t, i}, \underline{a}_{t,-i}\right)$. Similarly, the notation $\mathcal{A}_{-i}(t)=\prod_{j \neq i} \mathcal{A}_{j}(t)$ refers to the set of possible actions of all the player other than player $i$ at stage $t$ of the game. Let $\underline{h}_{t}=(\boldsymbol{\omega}(1), \underline{a}(1), \ldots, \underline{a}(t-1), \boldsymbol{\omega}(t))^{\mathbf{T}}$ be the history of the game at stage $t$ that lies in the set:

$$
\mathcal{H}_{t}=\left(\bigotimes_{t^{\prime}=1}^{t-1} \Omega \times \mathcal{A}\left(t^{\prime}\right)\right) \times \Omega .
$$

The utility function $\mathcal{U}_{i}$ for player $i$ is defined as:

$$
\mathcal{U}_{i}: \mid \begin{array}{ccc}
\mathcal{A}(t) \times \mathcal{H}_{t} & \longrightarrow & \mathbb{R} \\
\left(\underline{a}_{t}, \underline{h}_{t}\right) & \longmapsto & \mathcal{U}_{i}\left(\underline{a}_{t}, \underline{h}_{t}\right),
\end{array}
$$

which can be written as $\mathcal{U}_{i}\left(\underline{a}_{t, i}, \underline{a}_{t,-i}, \underline{h}_{t}\right)$. As all utilities depends on the game history $\underline{h}_{t}$, such argument is dropped in the rest of this paper, i.e., the utility of player $i$ for the action $\underline{a}_{t}$ is written as $\mathcal{U}_{i}\left(\underline{a}_{t}\right)$.

2) Potential Games: Potential games are introduced in [30]. The definition of a potential game $\mathcal{G}$ is given by:

Definition 3. The game $\mathcal{G}$ is a potential game if there exists a function $\phi$ such that for all player $i$ and actions $\underline{a}_{t}$ and $\underline{a}_{t}^{\prime}$ :

$$
\mathcal{U}_{i}\left(\underline{a}_{t}\right)-\mathcal{U}_{i}\left(\underline{a}_{t, i}^{\prime}, \underline{a}_{t,-i}\right)=\phi\left(\underline{a}_{t}\right)-\phi\left(\underline{a}_{t, i}^{\prime}, \underline{a}_{t,-i}\right) .
$$

In other words, a game $\mathcal{G}$ is a potential game if there is a function $\phi$ that measures exactly the difference in the utility due to a unilateral deviation by each player [29].

Such function $\phi$ is called the potential of the game that can be seen as a quantification of the disagreement among the players [30].

3) Equilibrium Existence and Pareto Optimality: The definition of a Nash Equilibrium (NE) is the following: 
Definition 4. An action profile $\underline{a}_{t}^{*} \in \mathcal{A}(t)$ is called a Nash equilibrium if the following holds for any player $i$ :

$$
\mathcal{U}_{i}\left(\underline{a}_{t}^{*}\right)=\max _{\underline{a}_{t, i} \in \mathcal{A}_{i}(t)} \mathcal{U}_{i}\left(\underline{a}_{t, i}, \underline{a}_{t,-i}^{*}\right) .
$$

In other words, an NE is an action profile $\underline{a}_{t}^{*}$ in which no player can increase his utility by unilateral deviation.

An attractive property of the NE is called the ParetoOptimum Nash Equilibrium (PONE) defined as follows:

Definition 5. An action profile $\underline{a}_{t}^{*} \in \mathcal{A}(t)$ is called a PONE if for any $N E$ action profile $\underline{b}_{t}^{*}$ and any player $i$ :

$$
\mathcal{U}_{i}\left(\underline{a}_{t}^{*}\right) \geq \mathcal{U}_{i}\left(\underline{b}_{t}^{*}\right) \text {. }
$$

In other words, the PONE is the NE that achieves the highest utility for all the players among all other NEs.

Before stating the main results concerning equilibria of potential games, first define the coordination game [31] as follows:

Definition 6. Let $\mathcal{G}=\left(\mathcal{M},\left\{\overline{\mathcal{A}}_{i}\right\}, \Omega,\left\{\mathcal{A}_{i}\right\},\left\{\alpha_{i}\right\},\left\{\mathcal{U}_{i}\right\}\right)$ be a potential game with a potential function $\phi$. The game $\mathcal{G}^{\prime}=$ $\left(\mathcal{M},\left\{\overline{\mathcal{A}}_{i}\right\}, \Omega,\left\{\mathcal{A}_{i}\right\},\left\{\alpha_{i}\right\}, \phi\right)$ is the coordination game of $\mathcal{G}$.

The following lemma links the equilibria of a potential game to the ones of its associated coordination game:

Lemma 1. Let $\mathcal{G}$ be a potential game with potential $\phi$ and $\mathcal{G}^{\prime}$ its associated coordination game. The set of NEs of $\mathcal{G}$ coincides with the set of NEs of $\mathcal{G}^{\prime}$. Moreover, the actions profile $\underline{a}_{t}^{*} \in \mathcal{A}(t)$ maxima of $\phi$ are $N E$ of $\mathcal{G}$.

Proof: The proof can be found in [31].

Note that the converse of this lemma is not true, i.e., not all the NEs of $\mathcal{G}$ are maxima of $\phi$ [31]. However, the existence of an NE is ensured by the following corollary:

Corollary 2. A finite potential game admits at least one NE.

Proof: The proof is a direct consequence of Lemma 1. For a finite game, the potential function is finite and, thus, has at least one maximum. Therefore, the game admits at least one NE.

The following theorem characterizes the PONE in a coordination game:

Theorem 1. Let $\mathcal{G}$ be a potential game with potential $\phi$ such that its corresponding coordination game is itself, i.e., $\mathcal{G}=\mathcal{G}^{\prime}$. The maximum of $\phi$ is the PONE of $\mathcal{G}$.

Proof: According to Lemma 1, the maximum $\underline{a}_{t}^{*}$ of $\phi$ is a NE of $\mathcal{G}$ and since $\phi$ is the utility function of $\mathcal{G}$, therefore, $\underline{a}_{t}^{*}$ is a NE that yields the highest utility. More specifically $\underline{a}_{t}^{*}$ yields the highest utility among all the NEs of $\mathcal{G}$ and thus it is the PONE.

\section{B. Games Formulation}

In this subsection, the delays reduction problems are formulated as potential games by considering the delay as the natural cost in the formulation. At each stage of the game, each player has two possible actions: either he transmits, or he listens. Therefore, the action space of player $i$ at each stage $t$ of the game is $\mathcal{A}_{i}(t)=\left\{\right.$ transmit $\underline{\kappa}^{i}(t)$, remain silent $\}$. Note that the action space of the players are not symmetric since each player can transmit a different packet combination at each stage. Let $\underline{a}_{t}$ be the actions taken by all the users at the stage $t$ defined as follows:

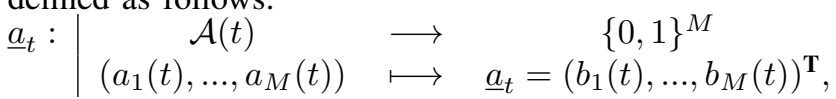

with $b_{i}(t)=0$ if $a_{i}(t)=\{$ remain silent $\}$ and 1 otherwise. The formulation of the completion time, maximum decoding delay and sum decoding delay games is the following:

- Players: Users in set $\mathcal{M}$

- History : $\underline{h}_{t}=$ Channel realization $\boldsymbol{\omega}(t)$ and players' action $\underline{a}_{t}$ at each stage $t \geq 1$.

- Strategies: Contingency plans for selection transmission policy at each stage $t \geq 1$ and for any given history $\underline{h}_{t}$.

- Utilities: Function $\mathcal{U}_{i}$ for each player $i$, at each stage $t \geq 1$, for any history $\underline{h}_{t}$ and an action profile $\underline{a}_{t}$.

The utilities of the games are designed using the objective function defined in (7), (8), and (9) as follows:

- Game 1 (Completion time): $\mathcal{U}_{i}^{C T}=-\left\|\underline{\mathcal{C}}\left(\underline{a}_{t}\right)\right\|_{\infty}$.

- Game 2 (Max decoding delay): $\mathcal{U}_{i}^{M D D}=-\left\|\underline{\mathbb{D}}\left(\underline{a}_{t}\right)\right\|_{\infty}$.

- Game 3 (Sum decoding delay): $\mathcal{U}_{i}^{S D D}=-\left\|\underline{\mathbb{D}}\left(\underline{a}_{t}\right)\right\|_{1}$.

The defined above games is a non-cooperative stochastic game [29] whose utilities does not depend on the player. Therefore, they belong to the potential game class.

\section{Game Equilibrium Analysis}

This subsection derives the expressions of the sets of NEs of the above-defined games. First introduce the set of actions $E(t)$ at stage $t$ of the game in which exactly one player or more than 2 players are transmitting, expressed as:

$$
E(t)=\left\{\underline{a}_{t} \in \mathcal{A}(t) \text { s.t. }\left\|\underline{a}_{t}\right\|_{1}=1 \text { or }\left\|\underline{a}_{t}\right\|_{1}>2\right\} .
$$

Let $Q(t, \alpha)$ be the set of players that are able to increase the maximum utility from its value at time $t-1$ if they experience $\alpha=\left\{\alpha_{1}, \cdots, \alpha_{M}\right\}$ unit of delay. The mathematical definition of is $Q(t, \alpha)$ is the following:

$$
Q(t)=\left\{i \in \mathcal{M} \text { s.t. } \mathcal{U}_{i}\left(\underline{a}_{t-1}\right)+\alpha_{i}>\max _{j \in \mathcal{M}} \mathcal{U}_{j}\left(\underline{a}_{t-1}\right)\right\},
$$

where the utility depends on the game under investigation. Further, let $Y_{0}(t, \alpha)$ be the increase in utility when not exactly one user is transmitting and $Y_{i}\left(t, \alpha_{i}\right)$ when only user $i$ is transmitting. Define $Z(t, \alpha)=\left\{i \in \mathcal{M}\right.$ s.t. $\left.Y_{i}\left(t, \alpha_{i}\right) \leq Y_{0}(t, \alpha)\right\}$ as the set of user that can maintain the maximum utility at its value. Finally, let $\tilde{E}(t, \alpha)$ be the action profiles in which two players $i$ and $j$ are transmitting and increasing the maximum utility defined as follows:

$$
\tilde{E}(t, \alpha)=\left\{\underline{a}_{t} \in \mathcal{A}(t) \text { s.t. }\left\|\underline{a}_{t}\right\|_{1}=2 \text { and } i, j \notin Z(t, \alpha)\right\} .
$$

The following theorem characterizes the NEs of the completion time game:

Theorem 2. The set of NEs of Game 1 can be expressed as follows:

$$
\mathcal{E}^{C T}(t)=\left\{\begin{array}{l}
\mathcal{A}(t) \text { if } Z(t, \alpha)=\varnothing \\
E(t) \cup \tilde{E}(t, \alpha) \text { otherwise },
\end{array}\right.
$$

with $\alpha_{i}=1 /\left(1-\tilde{p}_{i}\right)$.

Proof: The proof can be found in Appendix A.

In a similar manner, the set of NEs of game 2 are given in the following corollary: 
Corollary 3. The set of NEs of Game 2 can be expressed as follows:

$$
\mathcal{E}^{M D D}(t)=\left\{\begin{array}{l}
\mathcal{A}(t) \text { if } Z(t, \alpha)=\varnothing \\
E(t) \cup \tilde{E}(t, \alpha) \text { otherwise }
\end{array}\right.
$$

with $\alpha_{i}=1$.

Proof: The proof can be found in Appendix B.

The set of NEs of the sum decoding delay game is given in the following theorem:

Theorem 3. The set of NEs of Game 3 is $\mathcal{E}^{S D D}(t)=E(t)$

Proof: The proof can be found in Appendix C.

These games, even though well-defined for systems with perfect and complete information (i.e., all the information in the system are common knowledge by all players), suffer from many flaws in systems with incomplete information. First, by inspection of the NEs of the games, it is clear that most of the NEs yield the worst payoff. In all these game formulations, any action profile $\underline{a}_{t} \in \mathcal{A}(t)$ such that $\left\|\underline{a}_{t}\right\|_{1}>2$ is a NE of the game. The number of such actions is $\left(\begin{array}{c}M \\ 3\end{array}\right)$ compared to the $M$ actions profile of interest (i.e., action profiles in which only one player is transmitting). Secondly, in incomplete information scenario, such games may loop infinitely without reducing the Wants set of any player as they can operate in one of the non-desired NE points. For these reasons, the overall performance of the games is destitute in the incomplete information scenario. The paper proposes, in the next section, to address the robustness of the formulations by proposing less sensitive utilities based on a punishment policy.

\section{Punishment And Bargaining}

This section first suggests a punishment policy to prevent the occurrence of multiple packet collisions in a D2D enabled network. Such system allows the reformulation of the delays reduction problem as Nash bargaining problems so as to limit the set of NEs to the actions of interest, i.e., those that reduce a Wants set of at least one of the players. The newly designed utilities are then shown to be more robust to incomplete information and to provide more efficient and stable solutions.

\section{A. Punishment and Back-off Function}

In single-hop transmissions, simultaneous communications result in packet collision and interference at all players. Mechanisms for collision detection are inherent to the physical layer design and abstracted in this paper as a one-bit hard decision indication the collision event.

In the previous games design, players responsible for collisions are allowed to transmit in subsequent time slots. In order to overcome scenarios in which multiple consecutive collisions take place, a punishment period of $V$ time slots is imposed on the responsible players. In other words, during the following $V$ transmissions such players back-off. Let $\underline{c}_{t}=\left(c_{1}(t), \ldots, c_{M}(t)\right)^{\mathbf{T}} \in\{0,1\}^{M}$ be the collision indicator that takes the value 1 if player $i$ is accountable for a collision at time $t$. Let $\mathbf{C}$ be the collision history of the last $V$ stage of the game. The mathematical definition of this variable is:

$$
\mathbf{C}: \mid \begin{array}{llc}
\mathcal{H}_{t} & \longrightarrow & \{0,1\}^{M \times V} \\
\underline{h}_{t} & \longmapsto & \mathbf{C}\left(\underline{h}_{t}\right)=\left[\underline{c}_{t-V}, \ldots, \underline{c}_{t-1}\right] .
\end{array}
$$

The back-off function $\underline{\mathcal{B}}$ indicating, at each stage $t$ of the game, which players are allowed to transmit, can expressed as $\underline{\mathcal{B}}\left(\underline{h}_{t}\right)=\mathbf{C}\left(\underline{h}_{t}\right) \underline{1}$. A zeros-value of the $i$-th element of $\underline{\mathcal{B}}$ indicates that the $i$-th player is allowed to transmit and 1 otherwise.

\section{B. Games Formulation}

The Nash bargaining solution is a solution concept introduced by Nash initially for the two-player games. One essential idea of the bargaining theory is that solution notions of cooperative games can be interpreted as non-cooperative games based on individual utility maximization from the knowledge/belief of the players. The construction of noncooperative bargaining games that sustain multiple cooperative solution concepts as their equilibrium outcomes is mainly based on the idea of having a solution that is fair and more efficient than the one obtained without bargaining or agreement. The bargaining solution is characterized by its Pareto optimality, individual rationality, invariance to positive affine transformation, independence of irrelevant alternatives and symmetry. The following theorem links the bargaining solution to the Pareto-optimal action profile:

Theorem 4. For games with unique Pareto-optimal action profile, they can be reformulated such that there exists a unique Nash bargaining action verifying the axioms of the bargaining solution. Such solution is the unique Pareto-optimal action profile.

Proof: The proof can be found in [29].

According to Theorem 1, the Pareto-optimal action profile of the proposed games is the PONE. Therefore, from Theorem 4 if the PONE of the game is unique, the bargaining solution is the PONE of the game. In order to fulfill that previous condition, this subsection reformulates the games as follows:

- Game 4 (Completion time):

$$
\mathcal{U}_{i}^{C T}\left(\underline{a}_{t}\right)=-\left\|\underline{\mathcal{C}}\left(\underline{a}_{t}\right)\right\|_{\infty}-\left\|\underline{a}_{t}\right\|_{1}-\gamma\left(\underline{a}_{t}\right) .
$$

- Game 5 (Max decoding delay):

$$
\mathcal{U}_{i}^{M D D}\left(\underline{a}_{t}\right)=-\left\|\underline{\mathbb{D}}\left(\underline{a}_{t}\right)\right\|_{\infty}-\left\|\underline{a}_{t}\right\|_{1}-\gamma\left(\underline{a}_{t}\right) .
$$

- Game 6 (Sum decoding delay):

$$
\mathcal{U}_{i}^{S D D}\left(\underline{a}_{t}\right)=-\left\|\underline{\mathbb{D}}\left(\underline{a}_{t}\right)\right\|_{1}-\left\|\underline{a}_{t}\right\|_{1},
$$

where $\gamma\left(\underline{a}_{t}\right)=\left(\left\|\underline{\mathbb{D}}\left(\underline{a}_{t}\right)-\underline{\mathbb{D}}(t-1)\right\|_{1}\right) / M$.

In these reformulations of the games, sparsity of the action profile is encouraged by including the $\ell_{1}$ regularizer. Moreover, $\gamma$ prioritizes players when all action profiles yield a higher utility than in the previous stage of the game. This additional term is scaled by the number of players so as to maintain the ordinal action preference of the original games. It can be easily seen that Games 4, 5, and 6 are non-cooperative stochastic potential games. Further, next subsection shows that the set of NEs is reduced to the one-dimensional line in which only one player is transmitting containing the Nash bargaining solution of interest. 


\section{Equilibrium Stability}

In virtue of Corollary 2, all previously defined games admit at least one NE. Moreover, according to Theorem 1, the maximum of the utility function is the PONE of the game. However, the existence of NEs and/or the PONE is not sufficient to guarantee the robustness of the formulations. One popular metric, introduced in [32], to characterize the equilibria of a game is the price of anarchy defined as follows:

Definition 7. The price-of-anarchy (PoA) is defined as the worst-case efficiency of a Nash Equilibrium among all possible strategies. Such PoA can be expressed, at stage t of the game, as follows:

where:

$$
P o A(t)=\frac{\max _{s \in S(t)} W(s)}{\min _{s \in \mathcal{E}(t)} W(s)},
$$

- $S(t)$ is the set of all possible strategies at stage $t$,

- $\mathcal{E}(t)$ is the set of all NEs at stage $t$,

- $W: S \longrightarrow \mathbb{R}$ is a fairness function.

The PoA is a concept that measures how the efficiency of a game degrades due to the selfish behavior of its players. In the scenario considered in this paper, the Pareto Equilibrium is a Nash equilibrium, and the utility functions are the same for all the players, i.e., the scenario of Theorem 1. Such properties allow the reformulation of the PoA as follows:

$$
P o A(t)=\frac{\max _{s \in \mathcal{E}(t)} \phi(s)}{\min _{s \in \mathcal{E}(t)} \phi(s)} \text {. }
$$

Let $\mathcal{O}(t)=\left\{\underline{a}_{t} \in \mathcal{A}(t)\right.$ s.t. $\left.\left\|\underline{a}_{t}\right\|_{1}=1\right\}$ be the set of action profiles in which a single player is transmitting. The one-dimensional line $\mathcal{O}(t)$ contains only the useful operating points in which at least one player benefits from the transmissions. The following theorem characterizes the NEs and the robustness of the completion time game:

Theorem 5. The set of NEs of Game 4 is included in the one-dimensional line in which a single user is transmitting, i.e., $\mathcal{E}^{C T}(t) \subseteq \mathcal{O}(t)$. Furthermore, for the practical stages in which the anticipated completion time is not surely increasing, i.e., $Z(t, \alpha) \neq \varnothing, \alpha_{i}=1 /\left(1-\tilde{p}_{i}\right)$, the PoA of Game 4 (denoted by $\mathrm{PoA}_{4}$ ) is strictly more efficient than the one of Game 1 (denoted by $P o A_{1}$ ). In other words, $0 \leq P o A_{1}(t)<$ $\mathrm{PoA}_{4}(t) \leq 1$.

Proof: The proof can be found in Appendix D.

In a similar manner, the set of NEs of Game 5 are given in the following corollary:

Corollary 4. The set of NEs of Game 5 is included in the one-dimensional line in which a single user is transmitting, i.e., $\mathcal{E}^{M D D}(t) \subseteq \mathcal{O}(t)$. Furthermore, for the practical stages in which the maximum decoding delay is not surely increasing, i.e., $Z(t, 1) \neq \varnothing$, the PoA of Game 5 (denoted by PoA $A_{5}$ ) is strictly more efficient than the one of Game 2 (denoted by $\mathrm{PoA}_{2}$.

Proof: The proof can be found in Appendix E.

The set of NEs and the robustness of the sum decoding delay game is given in the following theorem:
Theorem 6. The set of NEs of Game 6 is equal to the onedimensional line in which a single user is transmitting, i.e., $\mathcal{E}^{S D D}(t)=\mathcal{O}(t)$. Furthermore, at any stage $t$, the PoA of Game 6 (denoted by $\mathrm{PoA}_{6}$ ) is more efficient than the one of Game 3 (denoted by $\mathrm{PoA}_{3}$ ).

Proof: The proof can be found in Appendix F.

\section{Distributed Learning Algorithms}

Popular assumptions about the players' knowledge in game theory are the complete and perfect information. The former assumption means that the actions available to the players and the utility functions are common knowledge, i.e., every player knows the data of the game, every player knows that the other players know the data of the game, every player knows that every player knows that every player knows the data of the game, and so on, ad infinitum. The latter assumption means all the players know the history of the game entirely. Note that these assumptions do not add extra constraints to the problem but are rather intrinsic to it for systems operating with full feedback.

Learning to play equilibrium is the strategies each player adopts and updates depending on his knowledge to attaining an equilibrium. While reaching the optimal solution for games with perfect and complete information is trivial, learning and dynamics are crucial under uncertain, time-variant, and dynamic environment. This section proposes distributed learning algorithms that operate at one of the equilibrium points of systems with either complete or incomplete information. The section further suggests that the proposed framework can be easily extensible to imperfect feedback scenarios and provides as an example a learning algorithm for the lossy feedback case. As the reformulations of the games are more robust than the originals versions, this section suggests learning algorithms only for games 4,5 , and 6 .

\section{A. Best Response Algorithm}

This paper proposes the best response algorithm to learning to play equilibrium in complete and perfect information scenario. In the original formulation of the best response algorithm, by Cournot [33], players choose their actions sequentially. At each time slot, a player selects the action that is the best response to the action chosen by the other players in the previous time slot. Since the state of the game $\omega \in \Omega$, i.e., the channel realization, is not known to players, the utility function is replaced by the expected utility function. This can be done by replacing the actual state $\underline{\omega}_{i}(t)$ by its expected value $\underline{P}_{i}(t)$ in (4). The following theorem characterizes the outcome of the best-response algorithm applied to the proposed games:

Theorem 7. The best-response algorithm operates the at the PONE of each of the proposed games with perfect and complete information.

Proof: To prove this theorem, introduce first the following lemma:

Lemma 2. Let $\mathcal{G}$ be a potential game with $\mathcal{V}$ a best response potential. If the action $\underline{a}_{t}^{*}$ maximizes $\mathcal{V}$, then $\underline{a}_{t}^{*}$ is a NE. 
Proof: The proof of this lemma can be found in [34].

From the previous analysis, the NEs of all games are included in the one-dimensional line in which only one player is transmitting. Let $\underline{a}_{t}^{*}$ be the PONE of the game such that $\left\|\underline{a}_{t}^{*}\right\|_{1}=a_{i}(t)=1$. Assume that the outcome of the bestresponse algorithm is the action profile $\underline{a}_{t}^{\prime} \neq \underline{a}_{t}^{*}$. In virtue of Lemma 2 and the equilibrium analysis in previous section, the action profile is a NE, i.e., $\left\|\underline{a}_{t}^{\prime}\right\|_{1}=a_{j}(t)=1, j \neq i$. For simplicity, assume that players take actions sequentially in order. Assume first that $j<i$, i.e., player $j$ takes action before player $i$. Therefore, player $j$ does not take its bestresponse game as he can ensure better payoff by choosing not to transmit. This results in $j \geq i$. Since player $i$ takes its best action, then he is transmitting. According to Lemma 2 player $j$ is unable to transmit otherwise the outcome is not an NE of the game. Finally, this concludes that $i=j$. In other words, the only outcome of the best-response algorithm is the PONE of the game.

In the setting mentioned above, every node computes the packet combination that other nodes can generate, allowing it to calculate the payoff function entirely. Therefore, the fully distributed system with perfect and complete information using the best-response algorithm yields the same performance as the centralized one without any additional signaling to coordinate the transmissions. However, the computation burden is reallocated to all mobile devices as they have to carry the same operation as the BS used to do in a centralized system. Next subsections relax such constraint by proposing learning algorithms for systems with incomplete information.

\section{B. Reinforcement Learning Algorithm}

This subsection suggests sacrificing optimality to reduce the computation load at each node by making less efficient decisions. Instead of performing all the calculations to determine the utility function, players may compute only their optimal packet combination. Although all the information is available at every node, the action set of other players is not shared knowledge between players and each player knows only its action set. Such assumption results in a game with incomplete information. In such scenario, this subsection suggests the reinforcement learning algorithm to learning to play equilibrium.

Reinforcement learning [35] is originally studied in the context of a single player games. The idea behind this algorithm is that the players interact with their environment and depending on the outcome of the past actions, they decide to choose or avoid certain actions. Actions that lead to a high (satisfactory) payoff by the past are repeated if the same situation occurs. Actions that are not satisfactory tend to be avoided. In a game theory context, reinforcement learning is implemented by associating a probability distribution over the set of possible actions for all players and update these distributions according to the perceived payoff at each stage of the game. This paper uses the reinforcement algorithm introduced by Bush and Mosteller [36] for stochastic games. The players select stochastically the actions to take according to their probabilities. Formally, the probability $x_{j}$ that player $j$ takes the action $s_{j}$ at stage $t+1$ is calculated as follows:

$x_{j, t+1}\left(s_{j}\right)= \begin{cases}x_{j, t}\left(s_{j}\right)+\lambda_{j} \bar{s}_{j, t}\left(1-x_{j, t}\left(s_{j}\right)\right) & \text { if } \bar{s}_{j, t} \geq 0 \\ x_{j, t}\left(s_{j}\right)+\lambda_{j} \bar{s}_{j, t} x_{j, t}\left(s_{j}\right) & \text { if } \bar{s}_{j, t}<0,\end{cases}$

where $\lambda_{j}$ is the learning rate of player $j\left(0<\lambda_{j}<1\right)$, and $\bar{s}_{j, t}$ is the stimulus of action $a_{i}\left(-1<\bar{s}_{j, t}<1\right)$ defined as follows:

$$
\bar{s}_{j, t}=\frac{\phi_{j}\left(a_{i}, t\right)-M_{j}}{\max _{a \in \mathcal{A}}\left|\phi_{j}(a, t)-M_{j}\right|},
$$

with $\phi_{j}(a, t)$ is the payoff perceived by player $j$ at time $t$ after taking the action $a$ and $M_{j}$ is a satisfactory level for player $j$.

For the potential games class, the reinforcement algorithm converges to an NE of the game with probability 1 [34] for any learning rate and satisfactory threshold. The optimal choice of the learning rate or the adequate threshold, typically, needs to be determined in an outer power optimization step, but this falls outside the scope of the current paper. This paper proposes the following fixed values for both variables: the learning rate of a player $i$ is the ratio of its Has set $\left|\mathcal{H}_{i}\right|$ by the total number of packets $N$ and the satisfactory criterion is $-\left|M_{w}\right|_{1}+\epsilon$ for some $\epsilon>0$. In other words, players are satisfied if at least one player is targeted, i.e., any action profile that does not yield the worst payoff.

For the proposed games, the reinforcement learning algorithm is fluctuating between transient and stable states. In the temporary state, players with the largest Has set are more likely to transmit. Should collisions occur, the responsible players back-off. The process is repeated until only one player communicates. The system, then, reaches a stable phase in which the player continues to send in the following stages until he becomes unable to satisfy any other player. The perceived payoff being no longer satisfactory, the system switches to the transient phase and the steps above are repeated.

\section{Imperfect Feedback Scenario}

This subsection suggests that the proposed framework can be easily extensible to imperfect feedback scenarios and provides as an example a learning algorithm for the lossy feedback case. In lossy feedback configuration, the feedback is subject to erasure at players that can create uncertainties in the feedback matrix and the overall system. In game theory terms, the lossy feedback situation mean that neither the information of the game nor the action sets are common knowledge between players.

Even though the uncertainties in the determination of the utility function, players can generate packet combination to reduce it. The expressions of these packet combinations can be found in [37] for the completion time and the maximum decoding delay reduction, and in [25] for the sum decoding delay minimization.

In order to learning to play equilibrium in such incomplete and imperfect information scenario, this subsection proposes a modified version of the reinforcement algorithm introduced in the previous subsection. Given that each player perfectly knows its Has set, then the proposed learning rate in the previous subsection is a well-defined quantity. However, the property does not hold for the stimulus since players do no have full access to the real payoff of their actions. The 


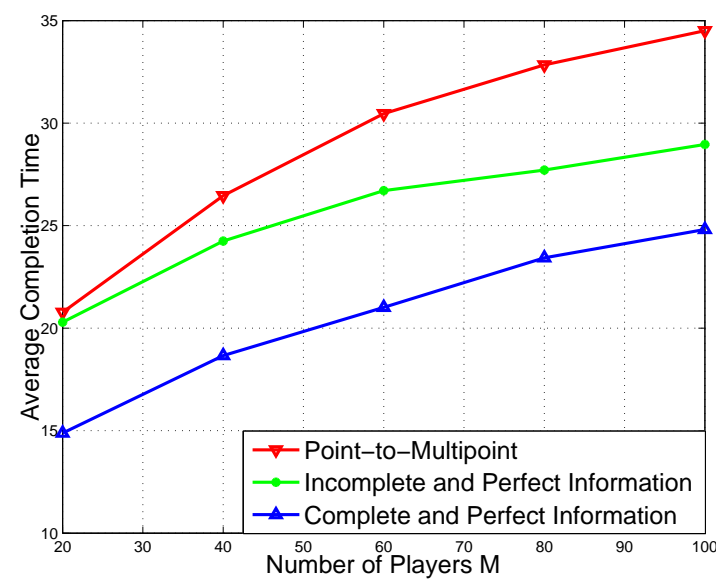

Fig. 1. Mean completion time versus number of players $M$ for a network composed of $N=30$ packets, an average player-to-player erasure $P=0.1$, and a sender-to-player erasure $Q=0.2$.

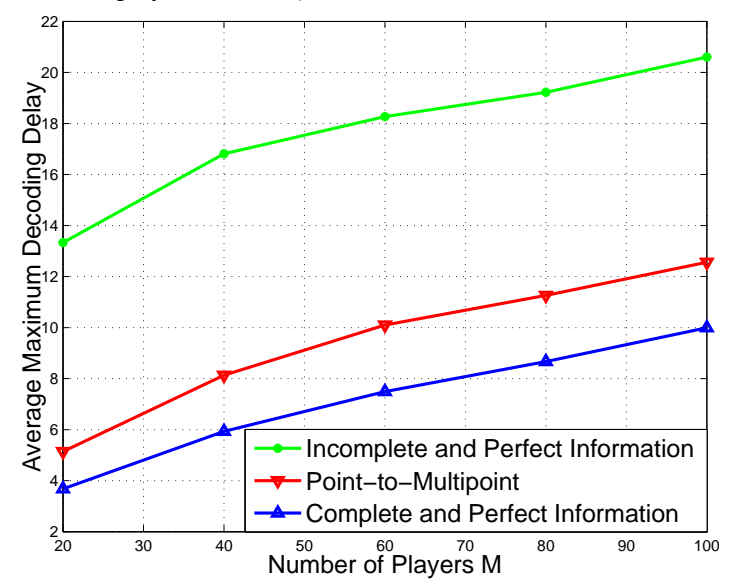

Fig. 2. Mean maximum delay versus number of players $M$ for a network composed of $N=30$ packets, an average player-to-player erasure $P=0.1$, and a sender-to-player erasure $Q=0.2$.

authors in [38] introduce a reinforcement learning consisting in updating the probability distribution of action $s_{j}$ to be taken in time $t+1$ as follows:

$$
x_{j, t+1}\left(s_{j}\right)=x_{j, t}\left(s_{j}\right)+\lambda_{j} \tilde{s}_{j, t}\left(\mathbb{1}_{\left\{a_{j, t=s_{j}}\right\}}-x_{j, t}\left(s_{j}\right)\right),
$$

where $\tilde{s}_{j, t}$ is an estimate of the payoff at stage $t$ of the game and $\mathbb{1}$ is the step function. Such estimated payoff is considered to be the expected value of the utility function determined when computing the packet combination of each of the delay aspects. Naturally, the learning rate and the estimated payoff need to be updated in an outer optimization loop. However, this falls out of the scope of the current paper.

\section{Simulation Results}

This section shows the performance of the proposed fully distributed solutions for perfect/imperfect and complete/incomplete information against the PMP setting for both accurate and lossy feedback scenarios. This section assumes a player-to-player erasure probability $P$ smaller than the senderto-player erasure $Q$ as the short range communications are more reliable than the base station-player communications [12], [24]. The section further proposes simulation against such parameter.

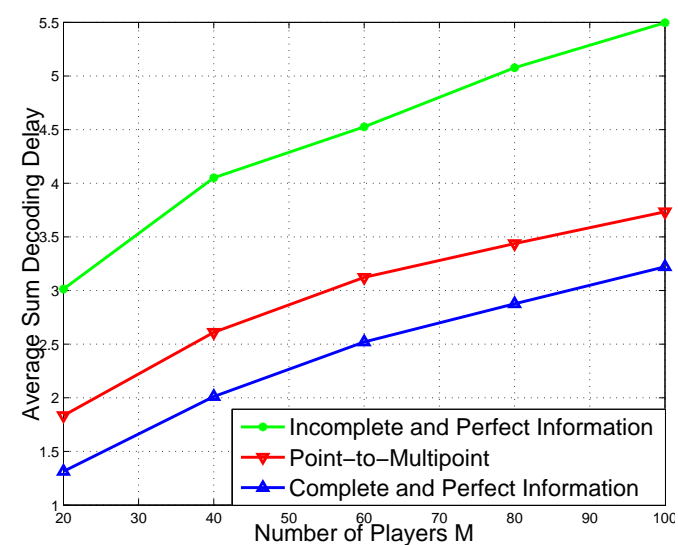

Fig. 3. Mean sum decoding delay versus number of players $M$ for a network composed of $N=30$ packets, an average player-to-player erasure $P=0.1$, and a sender-to-player erasure $Q=0.2$.

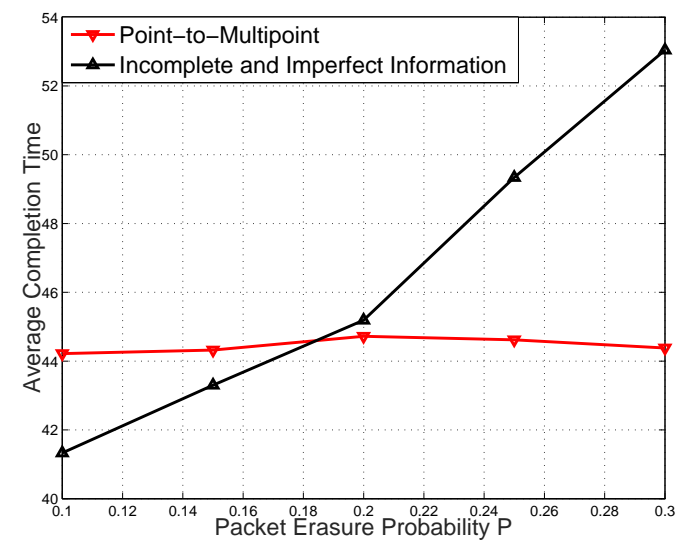

Fig. 4. Mean completion time in lossy feedback scenario versus playersto-player erasure probability $P$ for a network composed of $M=60$ player, $N=30$ packets, and a sender-to-player erasure $Q=0.3$.

In these simulations, the delay is computed over a large number of iterations, and the average value is presented. The packet erasure probability is assumed to remains constant during a delivery period and change from iteration to iteration while keeping its mean, $P$ of the player-player and $Q$ for the base BS-players, constant. Further, each player is assumed to have perfect knowledge of the packet erasure probabilities, i.e., the estimation of this probability is perfect.

Figure 1 depicts the comparison of the average completion time against the number of players $M$ in perfect feedback scenario for a network composed of $N=30, Q=0.2$, and $P=0.1$. Figure 2 and Figure 3 illustrate the comparison in perfect feedback scenario for the maximum decoding delay and the sum decoding delay, respectively, against the number of players $M$ for a network composed of $N=30, Q=0.2$, and $P=0.1$.

From all figures, it can explicitly be noted that the proposed cooperative algorithm with perfect and complete information outperforms the traditional PMP approach. From Figure 1, for a small number of players, the PMP setting is close to the proposed scheme with incomplete and perfect information. This can be explained by the fact that, for a small number of players, the probability that the union of the Has sets of all the players is equal to $\mathcal{N}$ is low. Thus, the BS carries a good portion of the recovery process until this condition occurs, and 


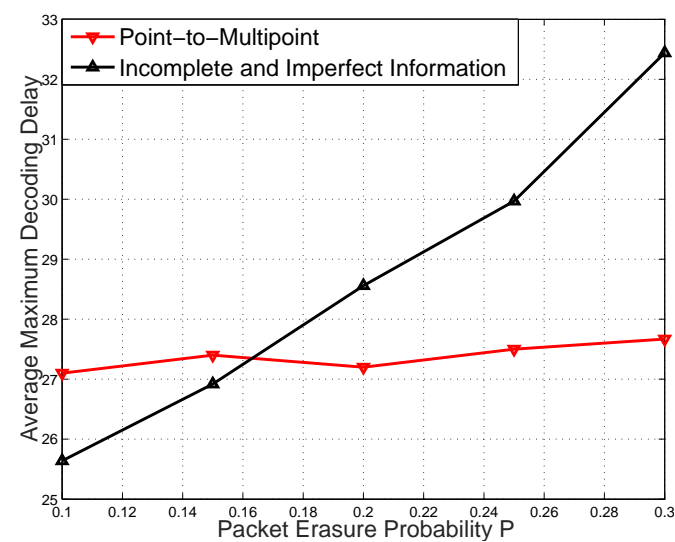

Fig. 5. Mean maximum delay in lossy feedback versus players-to-player erasure probability $P$ for a network composed of $M=60$ player, $N=30$ packets, and a sender-to-player erasure $Q=0.3$.

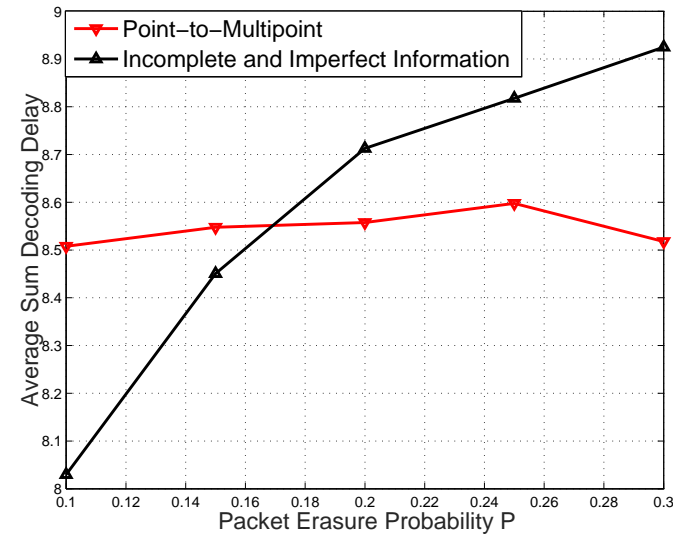

Fig. 6. Mean sum decoding delay in lossy feedback versus players-to-player erasure probability $P$ for a network composed of $M=60$ player, $N=30$ packets, and a sender-to-player erasure $Q=0.3$.

then D2D takes place. This makes the overall performance close to the absolute PMP scheme. However, the larger the number of players, the bigger the probability that the union of their Has sets is equal to $\mathcal{N}$ and thus the more D2D is employed, the greater the gap between the PMP performance.

Figure 2 and Figure 3 show that the proposed distributed solution with incomplete and perfect information does not present any gain as compared to the PMP solution for the decoding delay reduction. Whereas such policy offers a better completion time, its performance does not translate the decoding delay reduction. This can be explained by the fact that, unlike the PMP and the algorithm with perfect and complete information, such policy suffers from collisions. Whereas a collision increases the completion time by only a single unit, it increases the decoding delay by $\underline{M}$ units which degrades the performance of the policy.

Figure 4 plots the mean completion time against the playerplayer erasure probability $Q$ in lossy feedback scenario for a network composed of $M=60, N=30$, and $Q=0.3$. Figure 5 and Figure 6 depict, respectively, the maximum and the sum decoding delay for the same system input parameters.

It can be noted from all figures that the proposed solution with incomplete and imperfect information outperforms the PMP in the lossy feedback scenario for a good enough playerplayer channel. However, the distributed solution rapidly degrades as the number of uncertainties (i.e., player-player erasure probability) increases. This can be explained by the fact that for a little erasure, the uncertainties are relatively rare events. Therefore, the system is nearly equivalent to the incomplete and perfect information system with accurate feedback. However, as the erasure increases, the uncertainties also increases, and players are more likely to take a less efficient decision since the estimation degrades.

\section{CONCLUSION}

This paper proposes a game-theoretical framework for fully distributed delay minimization problem for instantly decodable network coding based device-to-device communications. Game theory is employed as a tool to improve the distributed solution by overcoming the need for a central controller or additional signaling in the system. The sessions are modeled by self-interested players in non-cooperative potential games whose utility functions are designed in such way that increasing individual payoff results in a collective behavior achieving both a desirable system performance and the Nash bargaining solution. Learning algorithms are proposed for systems with complete and incomplete information so as to achieve the equilibrium in a fully distributed fashion. The advantage of this formulation is that it can be easily extended. As an example, the paper proposes learning algorithm in the lossy feedback scenario. Numerical results suggest that the proposed gametheoretical formulation provides appreciable performance gain against the conventional point-to-multipoint, especially for reliable player-to-player channels.

\section{APPENDIX A \\ ProOF OF THEOREM 2}

This section computes the set of NEs of the completion time game. For simplicity, this proof considers the cost function $\phi=-\mathcal{U}^{C T}$ with the objective of minimizing. In order to derive such set, first reformulate the cost function as follows:

$$
\phi\left(\underline{a}_{t}\right)=\phi\left(\underline{a}_{t-1}\right)+\xi\left(\underline{a}_{t}\right)
$$

with $\xi\left(\underline{a}_{t}\right)=\left\|\underline{\mathcal{C}}\left(\underline{a}_{t}\right)\right\|_{\infty}-\left\|\underline{\mathcal{C}}\left(\underline{a}_{t-1}\right)\right\|_{\infty}$. Therefore, the action profile $\underline{a}_{t}$ at time instant $t$ only affects the second term $\xi$.

Let $Q(t)$ be the set of players that can potentially increase the cost function at stage $t$ of the game. The mathematical definition of this set is the following:

$Q(t)=\left\{i \in \mathcal{M}\right.$ s.t. $\left.\mathcal{C}_{i}\left(\underline{a}_{t-1}\right)+1 /\left(1-\tilde{p}_{i}\right)>\left\|\underline{\mathcal{C}}\left(\underline{a}_{t-1}\right)\right\|_{\infty}\right\}$. Clearly, if $Q(t)=\varnothing$, then any action profile $\underline{a}_{t}^{*} \in \mathcal{A}(t)$ is a NE since all the profiles do not change the cost function, i.e., $\phi\left(\underline{a}_{t}^{*}\right)=\phi\left(\underline{a}_{t-1}\right)$. In that case, the price of anarchy is $\operatorname{Po} A(t)=1$. Now assume $Q(t) \neq \varnothing$. For any action profiles $\underline{a}_{t}$ the cost function increases by the following quantity:

$$
\xi\left(\underline{a}_{t}\right)= \begin{cases}\max _{i \in Q(t)} \frac{1}{1-\tilde{p}_{i}} & \text { if }\left\|\underline{a}_{t}\right\|_{1} \neq 1 \\ \max _{i \in Q(t) \cap \underline{\mathcal{D}}\left(\underline{a}_{t}\right)} \frac{1}{1-\tilde{p}_{i}} & \text { if }\left\|\underline{a}_{t}\right\|_{1}=a_{j}(t)=1 .\end{cases}
$$

Let $Z(t)$ be the set of players that can target players in the critical set $Q(t)$ and reduce the increase in the cost function. Such set is defined as follows:

$$
Z(t)=\left\{j \in \mathcal{M} \text { s.t. } \max _{j \in Q(t) \cap \underline{\mathcal{D}}\left(\underline{a_{t}}\right)} \frac{1}{1-\tilde{p}_{j}}<\max _{i \in Q(t)} \frac{1}{1-\tilde{p}_{i}}\right\} .
$$


Two cases can be distinguished:

- $Z(t)=\varnothing$ : all action profile $\underline{a}_{t}^{*}$ yield the same cost function $\phi\left(\underline{a}_{t}^{*}\right)=\phi\left(\underline{a}_{t-1}\right)+\max _{i \in Q(t)} \frac{1}{1-\tilde{p}_{i}}$. Therefore all action profile are NEs of the game and the PoA is equal to 1 .

- $Z(t) \neq 0$ : action profile $\underline{a}_{t}^{*}=a_{j}(t)$ such that $j \in Z(t)$ yields a lower cost function than the other profiles.

Define $Y_{0}(t)=\max _{i \in Q(t)} \frac{1}{1-\tilde{p}_{i}}$ as the increase in the cost function when not exactly one player is transmitting and $Y_{j}(t)=\max _{i \in Q(t) \cap \underline{\mathcal{D}}\left(a_{t}\right)} \frac{1}{1-\tilde{p}_{i}}$ the increase when only player $j$ is transmitting. Clearly for action profiles $\underline{a}_{t}^{*}$ such that $\left\|\underline{a}_{t}^{*}\right\|_{1}=0$ are not NEs since the unilateral deviation of player $i \in Z(t)$ decreases the cost function. For action profile $\underline{a}_{t}^{*}$ such that $\left\|\underline{a}_{t}^{*}\right\|_{1}>2$, for any unilateral deviation, we have $\left\|\underline{a}_{t, i}, \underline{a}_{t,-i}^{*}\right\|>1$. Therefore, the cost function is unchanged and all these action profiles are NEs. Let $\underline{a}_{t}^{*}$ be an action profile such that $\left\|\underline{a}_{t}^{*}\right\|_{1}=a_{j}(t)=1$, then the difference in the cost function for any unilateral deviation of player is:

$$
\begin{aligned}
\phi\left(\underline{a}_{t, i}, \underline{a}_{t,-i}\right)-\phi\left(\underline{a}_{t}^{*}\right) & =\xi\left(\underline{a}_{t, i}, \underline{a}_{t,-i}^{*}\right)-\xi\left(\underline{a}_{t}^{*}\right) \\
& =Y_{0}(t)-Y_{j}(t) .
\end{aligned}
$$

Thus, any unilateral deviation yield the same or a higher cost function. These profiles are NEs of the game. Let the action profile be $\underline{a}_{t}^{*}$ with $\left\|\underline{a}_{t}^{*}\right\|_{1}=a_{i}(t)+a_{j}(t)=2$. Two scenarios can occur:

- $i \notin Z(t)$ and $j \notin Z(t)$ : By the same argument than for the case where $\left\|\underline{a}_{t}^{*}\right\|_{1}>2$, any unilateral deviation yields the same cost $\phi\left(\underline{a}_{t}^{*}\right)=\phi\left(\underline{a}_{t-1}\right)+Y_{0}(t)$. Therefore it is a $\mathrm{NE}$ of the game.

- $i \in Z(t)$ or $j \in Z(t)$ : By the same argument than for the case $\underline{a}_{t}^{*}=\underline{0}$, the cost function can be decreased by unilateral deviation of player $i$ if $j \in Z(t)$ and inversely. Such scenario is not a NE of the game.

Finally, the set of NEs of Game 1 can be expressed as follows:

$$
\mathcal{E}^{C T}(t)=\left\{\begin{array}{l}
\mathcal{A}(t) \text { if } Z(t)=\varnothing \\
E_{1}(t) \text { otherwise }
\end{array}\right.
$$

where the set $E_{1}(t)$ is defined as follows:

$$
\begin{aligned}
E_{1}(t) & =\left\{\underline{a}_{t} \in \mathcal{A}(t) \text { s.t. }\left\|\underline{a}_{t}\right\|_{1}=1 \text { or }\left\|\underline{a}_{t}\right\|_{1}>2\right. \\
& \text { or } \left.\left(\left\|\underline{a}_{t}\right\|_{1}=a_{i}(t)+a_{j}(t)=2 \text { and } i, j \notin Z(t)\right)\right\} .
\end{aligned}
$$

Substituting the above expression (A.3) in the PoA expression (25), for the practical stages in which $Z(t) \neq \varnothing$, the PoA of Game 1 can be expressed as follows:

$$
P o A_{1}(t)=1-\frac{Y_{0}(t)-\min _{j \in Z(t)}\left(Y_{j}(t)\right)}{\phi\left(\underline{a}_{t-1}\right)+Y_{0}(t)}
$$

\section{APPENDIX B}

\section{PROOF OF COROLlaRY 3}

This section computes the set of NEs of the maximum decoding delay game. For simplicity, this proof considers the cost function $\phi=-\mathcal{U}^{M D D}$ with the objective of minimizing. In order to derive such set, first reformulate the cost function as follows:

$$
\phi\left(\underline{a}_{t}\right)=\phi\left(\underline{a}_{t-1}\right)+\eta\left(\underline{a}_{t}\right),
$$

with the maximum decoding delay increase $\eta$ defined as follows:

$$
\eta\left(\underline{a}_{t}\right)= \begin{cases}1 & \text { if }\left\|\underline{\mathbb{D}}\left(\underline{a}_{t}\right)\right\|_{\infty}>\left\|\underline{\mathbb{D}}\left(\underline{a}_{t-1}\right)\right\|_{\infty} \\ 0 & \text { otherwise } .\end{cases}
$$

Therefore, the action profile $\underline{a}_{t}$ at time instant $t$ only affects the second term $\eta$. Let $Q(t)$ be the set of players that can potentially increase the cost function at stage $t$ of the game. The mathematical definition of this set is the following:

$$
Q(t)=\left\{i \in \mathcal{M} \text { s.t. } \mathbb{D}_{i}\left(\underline{a}_{t-1}\right)=\left\|\underline{\mathbb{D}}\left(\underline{a}_{t-1}\right)\right\|_{\infty}\right\} .
$$

Clearly, if $Q(t)=\varnothing$, then any action profile $\underline{a}_{t}^{*} \in \mathcal{A}(t)$ is a NE since all the profiles do not change the cost function, i.e., $\eta\left(\underline{a}_{t}\right)=0$. In that case, the price of anarchy is $\operatorname{Po} A(t)=1$. Now assume $Q(t) \neq \varnothing$. Let $q(t) \in\{0,1\}^{M}$ such that $q_{i}(t)=$ 1 iff $i \in Q(t)$. Two cases can be distinguished:

1) $\nexists i \in \mathcal{M}$ such that $\underline{\mathcal{D}}\left(\underline{a_{t}}\right) \circ \underline{q}(t)=\underline{0}$.

2) $\exists i \in \mathcal{M}$ such that $\underline{\mathcal{D}}\left(\underline{\overline{a_{t}}}\right) \circ \underline{q}(t)=\underline{0}$. Let $Z(t)$ be the set of such players.

In the first case, all action profile yield the same value of the cost $\eta\left(\underline{a}_{t}\right)=1$ and thus all of them are NEs of the game and $\operatorname{Po} A(t)=1$. In the second case, the cost varies with the chosen action profile. Let $\underline{a}_{t}^{*}$ be an action profile such that $\left\|\underline{a}_{t}^{*}\right\|_{1}>2$. For any unilateral deviation, we have $\left\|\left(\underline{a}_{t, i}, \underline{a}_{t,-i}^{*}\right)\right\|_{1}>1$. By definition of the delay $\underline{\mathbb{D}}$, we have:

$$
\underline{\mathbb{D}}\left(\underline{a}_{t, i}, \underline{a}_{t,-i}^{*}\right)=\underline{\mathbb{D}}\left(\underline{a}_{t-1}\right)+\underline{M}(t) .
$$

By assumption $Q(t) \neq \varnothing$, thus, the delay may be written as:

$$
\left\|\underline{\mathbb{D}}\left(\underline{a}_{t, i}, \underline{a}_{t,-i}^{*}\right)\right\|_{\infty}=\left\|\underline{\mathbb{D}}\left(\underline{a}_{t-1}\right)\right\|_{\infty}+1=\left\|\underline{\mathbb{D}}\left(\underline{a}_{t}^{*}\right)\right\|_{\infty} .
$$

Therefore, all action profile $\underline{a}_{t}^{*}$ with $\left\|\underline{a}_{t}^{*}\right\|_{1}>2$ are NEs of the game that yield the maximum value of the cost $\eta\left(\underline{a}_{t}\right)=$ 1. Consider now the action profile $\underline{a}_{t}^{*}=\underline{0}$, then for some deviation of any player $i \in Z(t)$, the cost decreases by the quantity:

$$
\phi\left(\underline{a}_{t}^{*}\right)-\phi\left(\underline{a}_{t, i}, \underline{a}_{t,-i}^{*}\right)=1, \forall \underline{a}_{t, i} \neq \underline{a}_{t, i}^{*} \in \mathcal{A}_{i}(t) .
$$

Therefore, the action profile of this type are not NEs of the game. Let the action profile be $\underline{a}_{t}^{*}$ with $\left\|\underline{a}_{t}^{*}\right\|_{1}=a_{i}(t)+$ $a_{j}(t)=2$. Two scenarios can occur:

- $i \notin Z(t)$ and $j \notin Z(t)$ : By the same argument than for the case where $\left\|\underline{a}_{t}^{*}\right\|_{1}>2$, any unilateral deviation yields the same cost $\phi\left(\underline{a}_{t}^{*}\right)=\phi\left(\underline{a}_{t-1}\right)+1$. Therefore it is a NE of the game.

- $i \in Z(t)$ or $j \in Z(t)$ : By the same argument than for the case $\underline{a}_{t}^{*}=\underline{0}$, the cost function can be decreased by unilateral deviation of player $i$ if $j \in Z(t)$ and inversely. This scenario is not a NE of the game.

Finally consider the action profile $\underline{a}_{t}^{*}$ with $\left\|\underline{a}_{t}^{*}\right\|_{1}=a_{i}(t)=1$. The following options may arise:

- $i \notin Z(t)$ : By the same argument than for the case where $\left\|\underline{a}_{t}^{*}\right\|_{1}>2$, any unilateral deviation yields the same cost $\eta\left(\underline{a}_{t}\right)=1$. Therefore it is a NE of the game.

- $i \in Z(t)$ : Any deviation increases the cost function. Hence, it is an NE of the game.

Finally, the set of NEs of Game 2 can be expressed as follows:

$$
\mathcal{E}^{M D D}(t)=\left\{\begin{array}{l}
\mathcal{A}(t) \text { if } Z(t)=\varnothing \\
E_{1}(t) \text { otherwise }
\end{array}\right.
$$


where the set $E_{1}(t)$ is defined as follows:

$$
\begin{aligned}
E_{1}(t) & =\left\{\underline{a}_{t} \in \mathcal{A}(t) \text { s.t. }\left\|\underline{a}_{t}\right\|_{1}=1 \text { or }\left\|\underline{a}_{t}\right\|_{1}>2\right. \\
& \text { or } \left.\left(\left\|\underline{a}_{t}\right\|_{1}=a_{i}(t)+a_{j}(t)=2 \text { and } i, j \notin Z(t)\right)\right\} .
\end{aligned}
$$

Substituting the above expression (B.7) in the PoA expression (25), for the practical stages in which $Z(t) \neq \varnothing$, the PoA of Game 2 can be expressed as follows:

$$
\operatorname{PoA}_{2}(t)=1-\frac{1}{\phi\left(\underline{a}_{t-1}\right)+1}
$$

\section{APPENDIX C \\ PROOF OF THEOREM 3}

This section computes the set of NEs of the sum decoding delay game. For simplicity, this proof considers the cost function $\phi=-\mathcal{U}^{S D D}$ with the objective of minimizing. In order to derive such set, first reformulate the cost function as follows:

$$
\phi\left(\underline{a}_{t}\right)=\phi\left(\underline{a}_{t-1}\right)+\psi\left(\underline{a}_{t}\right),
$$

with the sum decoding delay increase $\psi$ defined as follows:

$$
\psi\left(\underline{a}_{t}\right)= \begin{cases}\|\underline{M}(t)\|_{1} & \text { if }\left\|\underline{a}_{t}\right\|_{1} \neq 1 \\ \left\|\underline{\mathcal{D}}\left(\underline{a_{t}}\right)\right\|_{1} & \text { otherwise } .\end{cases}
$$

Therefore, the action profile $\underline{a}_{t}$ at time instant $t$ only affects the second term $\psi$. Let $\underline{a}_{t}^{*}$ be an action profile such that $\left\|\underline{a}_{t}^{*}\right\|_{1}>$ 2. For any unilateral deviation, we have $\left\|\left(\underline{a}_{t, i}, \underline{a}_{t,-i}^{*}\right)\right\|_{1}>1$. Therefore the cost function is unchanged by any unilateral deviation, i.e., $\phi\left(\underline{a}_{t, i}, \underline{a}_{t,-i}^{*}\right)=\phi\left(\underline{a}_{t}^{*}\right), \forall \underline{a}_{t, i} \in \mathcal{A}_{i}(t), \forall i \in \mathcal{M}$. Hence all action profile $\underline{a}_{t}^{*}$ such that $\left\|\underline{a}_{t}^{*}\right\|_{1}>2$ are NEs. These NEs yield the highest cost, i.e., $\psi\left(\underline{a}_{t}\right)=\|\underline{M}(t)\|_{1}$ among all possible action profiles.

Similarly, let $\underline{a}_{t}^{*}$ be an action profile such that $\left\|\underline{a}_{t}^{*}\right\|_{1}=$ $a_{j}(t)=1$. For any unilateral deviation, we have $\left\|\left(\underline{a}_{t, i}, \underline{a}_{t,-i}^{*}\right)\right\|_{1} \neq 1$. Therefore, the change in the cost function for any deviation $\underline{a}_{t, i} \in \mathcal{A}_{i}(t), \forall i \in \mathcal{M}$ is the following:

$$
\psi\left(\underline{a}_{t, i}, \underline{a}_{t,-i}^{*}\right)=\|\underline{M}(t)\|_{1}>\psi\left(\underline{a}_{t}^{*}\right)=\left\|\underline{\mathcal{D}}\left(\underline{a_{t}}\right)\right\|_{1},
$$

which concludes that all action profile $\underline{a}_{t}^{*}$ such that $\left\|\underline{a}_{t}^{*}\right\|_{1}=1$ are NEs. Now, let $\underline{a}_{t}^{*}=\underline{0}$, then any deviation decreases the cost by the quantity:

$$
\phi\left(\underline{a}_{t}^{*}\right)-\phi\left(\underline{a}_{t, i}, \underline{a}_{t,-i}^{*}\right)=\|\underline{M}(t)\|_{1}-\left\|\underline{\mathcal{D}}\left(\underline{a_{t}}\right)\right\|_{1}
$$

Clearly, these action profiles are not NE of the game. Finally, consider the action profile $\underline{a}_{t}^{*}$ such that $\left\|\underline{a}_{t}^{*}\right\|_{1}=a_{i}(t)+$ $a_{j}(t)=2$. The unilateral deviation of player $i$ or $j$ decreases the cost by the same quantity than in equation (C.4), which concludes that these type of action profile are not NEs of the game.

Finally, the set of NEs of Game 3 can be expressed as follows:

$$
\mathcal{E}^{S D D}(t)=\left\{\underline{a}_{t} \text { s.t. }\left\|\underline{a}_{t}\right\|_{1}=1 \text { or }\left\|\underline{a}_{t}\right\|_{1}>2\right\} .
$$

Substituting the above expression (C.5) in the PoA expression (25), the PoA of Game 3 can be expressed as follows:

$$
P o A_{3}(t)=\frac{\phi\left(\underline{a}_{t-1}\right)+\min _{\left\|\underline{a}_{t}\right\|_{1}=1}\left\|\underline{\mathcal{D}}\left(\underline{a}_{t}\right)\right\|_{1}}{\phi\left(\underline{a}_{t-1}\right)+\|\underline{M}(t)\|_{1}} .
$$

Furthermore, since $0 \leq\left\|\underline{\mathcal{D}}\left(\underline{a_{t}}\right)\right\|_{1} \leq\|\underline{M}(t)\|_{1}-1$, then the PoA can be bound by the following quantities:

$$
1-\beta^{-1}\|\underline{M}(t)\|_{1} \leq \operatorname{PoA}_{3}(t) \leq 1-\beta^{-1},
$$

where $\beta=\phi\left(\underline{a}_{t-1}\right)+\|\underline{M}(t)\|_{1}$ is the worst possible outcome.

\section{APPENDIX D \\ PROOF OF THEOREM 5}

In order to prove that the reformulation provides a more robust equilibria, this section first computes the set of NEs of the reformulation of the completion time game. For simplicity, this proof considers the cost function $\phi=-\mathcal{U}^{C T}$, defined in (21), with the objective of minimizing. The set of NEs allows the determination of the PoA of Game 4 and its comparison with the PoA of Game 1. In order to derive such set of NEs, first reformulate the cost function as follows:

$$
\phi\left(\underline{a}_{t}\right)=\phi\left(\underline{a}_{t-1}\right)+\xi\left(\underline{a}_{t}\right),
$$

with $\xi\left(\underline{a}_{t}\right)=\left\|\underline{a}_{t}\right\|_{1}+\gamma\left(\underline{a}_{t}\right)+\left\|\underline{\mathcal{C}}\left(\underline{a}_{t}\right)\right\|_{\infty}-\left\|\underline{\mathcal{C}}\left(\underline{a}_{t-1}\right)\right\|_{\infty}$. Therefore, the action profile $\underline{a}_{t}$ at time instant $t$ only affects the second term $\xi$.

Let $Q(t)$ be the set of players that can potentially increase the expected completion time at stage $t$ of the game. The mathematical definition of this set is the following:

$Q(t)=\left\{i \in \mathcal{M}\right.$ s.t. $\left.\mathcal{C}_{i}\left(\underline{a}_{t-1}\right)+1 /\left(1-\tilde{p}_{i}\right)>\left\|\underline{\mathcal{C}}\left(\underline{a}_{t-1}\right)\right\|_{\infty}\right\}$. Assume $Q(t)=\varnothing$, then any action profile $\underline{a}_{t}^{*} \in \mathcal{A}(t)$ does not increase the anticipated completion time, i.e., $\left\|\underline{\mathcal{C}}\left(\underline{a}_{t}\right)\right\|_{\infty}-$ $\left\|\underline{\mathcal{C}}\left(\underline{a}_{t-1}\right)\right\|_{\infty}=0$. Therefore, the increase in the cost function $\xi$ can be expressed as follows:

$$
\begin{aligned}
\xi\left(\underline{a}_{t}\right) & =\left\|\underline{a}_{t}\right\|_{1}+\gamma\left(\underline{a}_{t}\right) \\
& = \begin{cases}1+\frac{\left\|\underline{\mathcal{D}}\left(\underline{a}_{t}\right)\right\|_{1}}{M} & \text { if }\left\|\underline{a}_{t}\right\|_{1}=1 \\
\left\|\underline{a}_{t}\right\|_{1}+\frac{\|\underline{M}(t)\|_{1}}{M} & \text { otherwise . }\end{cases}
\end{aligned}
$$

Clearly, all the profiles $\underline{a}_{t}^{*}$ with $\left\|\underline{a}_{t}^{*}\right\|_{1}>1$ or $\left\|\underline{a}_{t}^{*}\right\|_{1}=0$ are not anymore NEs of the game. Therefore, if $Q(t)=\varnothing$, then the only NEs of the games are action profiles $\underline{a}_{t}$ such that $\left\|\underline{a}_{t}\right\|=1$. Now assume $Q(t) \neq \varnothing$ and let $Z(t)$ be the set of players that can target players in the critical set $Q(t)$ and reduce the increase in the cost function. This set is defined as:

$$
Z(t)=\left\{j \in \mathcal{M} \text { s.t. } \max _{i \in Q(t) \cap \underline{\mathcal{D}}\left(\underline{a_{t}}\right)} \frac{1}{1-\tilde{p}_{i}}<\max _{i \in Q(t)} \frac{1}{1-\tilde{p}_{i}}\right\} .
$$

Two cases can be distinguished:

- $Z(t)=\varnothing$ : the expected completion time increases by the same amount, i.e., $\left\|\underline{\mathcal{C}}\left(\underline{a}_{t}\right)\right\|_{\infty}-\left\|\underline{\mathcal{C}}\left(\underline{a}_{t-1}\right)\right\|_{\infty}=$ $\max _{i \in Q(t)} \frac{1}{1-\tilde{p}_{i}}$ for all action profiles.

- $Z(t) \neq 0$ : action profile $\underline{a}_{t}^{*}=a_{j}(t)$ such that $j \in Z(t)$ yields a lower completion time increase than the other profiles.

Define $Y_{0}(t)=\max _{i \in Q(t)} \frac{1}{1-\tilde{p}_{i}}$ as the increase in the completion time when not exactly one player is transmitting and $Y_{j}(t)=\max _{i \in Q(t) \cap \underline{\mathcal{D}}\left(\underline{a_{t}}\right)} \frac{1}{1-\tilde{p}_{i}}$ the increase when only player $j$ is transmitting. If $\bar{Z}(t)=\varnothing$ for an action profile $\underline{a}_{t}^{*}$, the cost function can be expressed as:

$$
\xi\left(\underline{a}_{t}^{*}\right)=Y_{0}(t)+ \begin{cases}\frac{\|\underline{M}(t)\|_{1}}{M} & \text { if }\left\|\underline{a}_{t}\right\|_{1}=0 \\ 1+\frac{\left\|\underline{\mathcal{D}}\left(\underline{a_{t}}\right)\right\|_{1}}{M} & \text { if }\left\|\underline{a}_{t}\right\|_{1}=1 \\ \left\|\underline{a}_{t}\right\|_{1}+\frac{\|\underline{M}(t)\|_{1}}{M} & \text { otherwise } .\end{cases}
$$


Clearly action profiles $\underline{a}_{t}^{*}$ of type $\left\|\underline{a}_{t}^{*}\right\|_{1} \neq 1$ are no more NEs of the game. Now assume $Z(t) \neq \varnothing$. Let $a_{t}^{*}$ be an action profile such that $\left\|\underline{a}_{t}^{*}\right\|_{1}>1$. For some unilateral deviation of player $i$ that is transmitting, the cost function decreases. In other words, we have:

$$
\begin{aligned}
& \phi\left(\underline{a}_{t, i}, \underline{a}_{t,-i}^{*}\right)-\phi\left(\underline{a}_{t}^{*}\right)= \\
& \left\{\begin{array}{c}
1>0 \quad \text { if }\left\|\underline{a}_{t, i}, \underline{a}_{t,-i}^{*}\right\|_{1}>1 \\
Y_{0}(t)-Y_{i}(t)+1+\frac{\|\underline{M}(t)\|_{1}-\left\|\underline{\mathcal{D}}\left(\underline{a_{t}}\right)\right\|_{1}}{M}>0 \\
\text { if }\left\|\underline{a}_{t, i}, \underline{a}_{t,-i}^{*}\right\|_{1}=1 \text { and } i \in Z(t) \\
1+\frac{\|\underline{M}(t)\|_{1}-\left\|\underline{\mathcal{D}}\left(\underline{a_{t}}\right)\right\|_{1}}{M}>0 \\
\text { if }\left\|\underline{a}_{t, i}, \underline{a}_{t,-i}^{*}\right\|_{1}=1 \text { and } i \notin Z(t) .
\end{array}\right.
\end{aligned}
$$

Thus, all action profile $\underline{a}_{t}^{*}$ with $\left\|\underline{a}_{t}^{*}\right\|_{1}>1$ are not NEs and it is clear to see that action profile $\underline{a}_{t}^{*}$ such that $\left\|\underline{a}_{t}^{*}\right\|_{1}=0$ are also not NEs of the game. Let $\underline{a}_{t}^{*}$ be an action profile such that $\left\|\underline{a}_{t}^{*}\right\|_{1}=a_{i}(t)=1$ and $i \notin Z(t)$. The difference in the cost when player $i$ deviates is:

$$
\phi\left(\underline{a}_{t}^{*}\right)-\phi\left(\underline{a}_{t, i}, \underline{a}_{t,-i}^{*}\right)=1+\frac{\left\|\underline{\mathcal{D}}\left(\underline{a}_{t}\right)\right\|_{1}-\|\underline{M}(t)\|_{1}}{M}>0 .
$$

Therefore such action profile is not a NEs. Now consider the action profile $\underline{a}_{t}^{*}$ such that $\left\|\underline{a}_{t}^{*}\right\|_{1}=a_{i}(t)=1$ and $i \in Z(t)$. The difference in the utility if any player deviates:

$$
\begin{gathered}
\phi\left(\underline{a}_{t, i}, \underline{a}_{t,-i}^{*}\right)-\phi\left(\underline{a}_{t}^{*}\right)= \\
\left\{\begin{array}{c}
1+Y_{0}(t)-Y_{i}(t)+\frac{\|\underline{M}(t)\|_{1}-\left\|\underline{\mathcal{D}}\left(\underline{a_{t}}\right)\right\|_{1}}{M}>0 \\
\text { if } \| \underline{a}_{t, j}, \underline{a_{t,-j}^{*} \|_{1}=2} \\
Y_{0}(t)-Y_{i}(t)+\frac{\|\underline{M}(t)\|_{1}-\left\|\underline{\mathcal{D}}\left(\underline{a_{t}}\right)\right\|_{1}}{M}>0 \\
\text { if }\left\|\underline{a}_{t, j}, \underline{a}_{t,-j}^{*}\right\|_{1}=0 .
\end{array}\right.
\end{gathered}
$$

The set of all NEs of the Game 4 can be expressed as:

$$
\mathcal{E}^{C T}(t)= \begin{cases}\mathcal{O}(t) \cap Z(t) & \text { if } Z(t) \neq \varnothing \\ \mathcal{O}(t) & \text { otherwise }\end{cases}
$$

where the notation $\mathcal{O}(t) \cap Z(t)$ refers to the set of action profiles $\underline{a}_{t}$ such that $\left\|\underline{a}_{t}\right\|_{1}=a_{i}(t)=1$ and $i \in Z(t)$. Substituting the above expression (D.5) in the PoA expression (25) for the practical stages in which the anticipated completion time is not surely increasing, i.e., $Z(t) \neq \varnothing$, the PoA of Game 4 can be expressed as follows:

$$
P o A_{4}(t)=\frac{\min _{\left\|\underline{a}_{t}\right\|_{1}=1} \phi\left(\underline{a}_{t-1}\right)+\frac{\left\|\underline{\mathcal{D}}\left(\underline{a_{t}}\right)\right\|_{1}}{M}+1+Y_{i}(t)}{\max _{\left\|\underline{a}_{t}\right\|_{1}=1} \phi\left(\underline{a}_{t-1}\right)+\frac{\left\|\underline{\mathcal{D}}\left(\underline{a}_{t}\right)\right\|_{1}}{M}+1+Y_{i}(t)}
$$

Moreover, since $0 \leq\left\|\underline{\mathcal{D}}\left(\underline{a_{t}}\right)\right\|_{1}<M$ for $\left\|\underline{a}_{t}\right\|_{1}=$, the PoA can be bounded by the following expressions:

$$
1 \geq \operatorname{PoA}_{4}(t)>1-\frac{1+Y_{0}(t)-\min _{j \in Z(t)}\left(Y_{j}(t)\right)}{\phi\left(\underline{a}_{t-1}\right)+2+Y_{0}(t)}
$$

Using the expression of the PoA of Game 1 in (A.4), it can be quickly concluded that for the practical stages in which the anticipated completion time is not inevitably increasing, i.e., $Z(t) \neq \varnothing$, the PoA of Game 4 is strictly more efficient than the one of Game 1. In other words, $0 \leq P_{o} A_{1}(t)<$ $\mathrm{PoA}_{4}(t) \leq 1$.

\section{APPENDIX E \\ PROOF OF COROLLARY 4}

In order to prove that the reformulation provides a more robust equilibria, this section first computes the set of NEs of the reformulation of the maximum decoding delay game. For simplicity, this proof considers the cost function $\phi=$ $-\mathcal{U}^{M D D}$, defined in (22), with the objective of minimizing. The set of NEs allows the determination of the PoA of Game 5 and its comparison with the PoA of Game 2. In order to derive such set of NEs, first reformulate the cost function as follows:

$$
\phi\left(\underline{a}_{t}\right)=\phi\left(\underline{a}_{t-1}\right)+\eta\left(\underline{a}_{t}\right)
$$

with the maximum decoding delay increase $\eta$ defined as follows:

$\eta\left(\underline{a}_{t}\right)=\left\|\underline{a}_{t}\right\|_{1}+\gamma\left(\underline{a}_{t}\right)+\left\{\begin{array}{l}1 \text { if }|| \underline{\mathbb{D}}\left(\underline{a}_{t}\right)\left\|_{\infty}>\right\| \underline{\mathbb{D}}\left(\underline{a}_{t-1}\right) \|_{\infty} \\ 0 \text { otherwise } .\end{array}\right.$

Let $Q(t)=\left\{i \in \mathcal{M}\right.$ s.t. $\left.\mathbb{D}_{i}\left(\underline{a}_{t-1}\right)=\left\|\underline{\mathbb{D}}\left(\underline{a}_{t-1}\right)\right\|_{\infty}\right\}$ be the set of players that can potentially increase the maximum decoding delay at stage $t$ of the game. Assume $Q(t)=\varnothing$, then any action profile $\underline{a}_{t}^{*} \in \mathcal{A}(t)$ does not increase the maximum decoding delay, i.e., $\left\|\underline{\mathbb{D}}\left(\underline{a}_{t}\right)\right\|_{\infty}-\left\|\underline{\mathbb{D}}\left(\underline{a}_{t-1}\right)\right\|_{\infty}=0$. Therefore, the increase in the cost function $\eta$ can be expressed as follows:

$$
\begin{aligned}
\eta\left(\underline{a}_{t}\right) & =\left\|\underline{a}_{t}\right\|_{1}+\gamma\left(\underline{a}_{t}\right) \\
& = \begin{cases}1+\frac{\left\|\underline{\mathcal{D}}\left(\underline{a}_{t}\right)\right\|_{1}}{M} & \text { if }\left\|\underline{a}_{t}\right\|_{1}=1 \\
\left\|\underline{a}_{t}\right\|_{1}+\frac{\|\underline{M}(t)\|_{1}}{M} & \text { otherwise . }\end{cases}
\end{aligned}
$$

Clearly, we can see that all the profiles $\underline{a}_{t}^{*}$ with $\left\|\underline{a}_{t}^{*}\right\|_{1}>1$ or $\left\|\underline{a}_{t}^{*}\right\|_{1}=0$ are not any more NEs of the game. Therefore, if $Q(t)=\varnothing$, then the only NEs of the games are action profiles $\underline{a}_{t}$ such that $\left\|\underline{a}_{t}\right\|=1$. Now assume $Q(t) \neq \varnothing$ and define $Z(t)$ as the set of players that can transmit a combination such that the maximum decoding delay do not increase. The mathematical definition of the set $Z(t)$ can be found in Appendix B. If $Z(t)=\varnothing$ it is clear to see that only the action profile with $\left\|\underline{a}_{t}^{*}\right\|_{1}=1$ are NEs and yield the cost $\eta\left(\underline{a}_{t}\right)=2+\frac{\left\|\underline{\mathcal{D}}\left(\underline{a}_{t}\right)\right\|_{1}}{M}$.

Now assume $Z(t) \neq \varnothing$. Let $\underline{a}_{t}^{*}$ be an action profile such that $\left\|\underline{a}_{t}^{*}\right\|_{1}>1$. For some unilateral deviation of player $i$ that is transmitting, the cost function decreases. In other words, we have:

$$
\begin{aligned}
& \phi\left(\underline{a}_{t}^{*}\right)-\phi\left(\underline{a}_{t, i}, \underline{a}_{t,-i}^{*}\right)= \\
& \left\{\begin{array}{c}
1>0 \quad \text { if }\left\|\underline{a}_{t, i}, \underline{a}_{t,-i}^{*}\right\|_{1}>1 \\
2+\frac{\|\underline{M}(t)\|_{1}-\left\|\underline{\mathcal{D}}\left(\underline{a_{t}}\right)\right\|_{1}}{M}>0 \\
\text { if }\left\|\underline{a}_{t, i}, \underline{a}_{t,-i}^{*}\right\|_{1}=1 \text { and } i \in Z(t) \\
M+\frac{\|\underline{M}(t)\|_{1}-\left\|\underline{\mathcal{D}}\left(\underline{a_{t}}\right)\right\|_{1}}{M}>0 \\
\text { if }\left\|\underline{a}_{t, i}, \underline{a}_{t,-i}^{*}\right\|_{1}=1 \text { and } i \notin Z(t) .
\end{array}\right.
\end{aligned}
$$

Thus, all action profile $\underline{a}_{t}^{*}$ with $\left\|\underline{a}_{t}^{*}\right\|_{1}>1$ are not NEs and it is clear to see that action profile $\underline{a}_{t}^{*}$ such that $\left\|\underline{a}_{t}^{*}\right\|_{1}=0$ are also not NEs of the game. Let $\underline{a}_{t}^{*}$ be an action profile such that $\left\|\underline{a}_{t}^{*}\right\|_{1}=a_{i}(t)=1$ and $i \notin Z(t)$. The difference in the 
cost when player $i$ deviates is:

$$
\phi\left(\underline{a}_{t}^{*}\right)-\phi\left(\underline{a}_{t, i}, \underline{a}_{t,-i}^{*}\right)=1+\frac{\left\|\underline{\mathcal{D}}\left(\underline{a}_{t}\right)\right\|_{1}-\|\underline{M}(t)\|_{1}}{M}>0 .
$$

Therefore such action profile is not a NEs. Now consider the action profile $\underline{a}_{t}^{*}$ such that $\left\|\underline{a}_{t}^{*}\right\|_{1}=a_{i}(t)=1$ and $i \in Z(t)$. The difference in the utility if any player deviates:

$$
\phi\left(\underline{a}_{t, i}, \underline{a}_{t,-i}^{*}\right)-\phi\left(\underline{a}_{t}^{*}\right)=\left\{\begin{array}{c}
2+\frac{\|\underline{M}(t)\|\left\|_{1}-\right\| \underline{\mathcal{D}}\left(\underline{a_{t}}\right) \|_{1}}{M}>0 \\
\text { if }\left\|\underline{\underline{a}} \underline{t}_{t, j}, \underline{a}_{t,-j}^{*}\right\|_{1}=0 \\
\frac{\left.\|\underline{M}(t)\|\left\|_{1}-\right\| \underline{\underline{a}}\right) \|_{1}>0}{M}>0 \\
\text { if }\left\|\underline{a}_{t, j}, \underline{a}_{t,-j}^{*}\right\|_{1}=2 .
\end{array}\right.
$$

The set of all NEs of the Game 5 can be expressed as:

$$
\mathcal{E}^{M D D}(t)= \begin{cases}\mathcal{O}(t) \cap Z(t) & \text { if } Z(t) \neq \varnothing \\ \mathcal{O}(t) & \text { otherwise }\end{cases}
$$

where the notation $\mathcal{O}(t) \cap Z(t)$ refers to the set of action profiles $\underline{a}_{t}$ such that $\left\|\underline{a}_{t}\right\|_{1}=a_{i}(t)=1$ and $i \in Z(t)$. Substituting the above expression (E.4) in the PoA expression (25) for the practical stages in which the anticipated completion time is not surely increasing, i.e., $Z(t) \neq \varnothing$, the PoA of Game 5 can be expressed as follows:

$$
P o A_{5}(t)=\frac{\min _{\left\|\underline{a}_{t}\right\|_{1}=1} \phi\left(\underline{a}_{t-1}\right)+\frac{\left\|\underline{\mathcal{D}}\left(\underline{a_{t}}\right)\right\|_{1}}{M}+1}{\max _{\left\|\underline{a}_{t}\right\|_{1}=1} \phi\left(\underline{a}_{t-1}\right)+\frac{\left\|\underline{\mathcal{D}}\left(\underline{a_{t}}\right)\right\|_{1}}{M}+1}
$$

Moreover, since $0 \leq\left\|\underline{\mathcal{D}}\left(\underline{a}_{t}\right)\right\|_{1}<M$ for $\left\|\underline{a}_{t}\right\|_{1}=$, the PoA can be bounded by the following expressions:

$$
1 \geq \operatorname{Po}_{5}(t)>1-\frac{1}{\phi\left(\underline{a}_{t-1}\right)+2}
$$

Using the expression of the PoA of Game 1 in (B.8), it can be quickly concluded that for the practical stages in which the anticipated completion time is not inevitably increasing, i.e., $Z(t) \neq \varnothing$, the PoA of Game 5 is strictly more efficient than the one of Game 2. In other words, $0 \leq P_{o} A_{2}(t)<$ $\operatorname{PoA}_{5}(t) \leq 1$.

\section{APPENDIX F \\ PROOF OF THEOREM 6}

In order to prove that the reformulation provides a more robust equilibria, this section first computes the set of NEs of the reformulation of the sum decoding delay game. For simplicity, this proof considers the cost function $\phi=-\mathcal{U}^{S D D}$, defined in (23), with the objective of minimizing. The set of NEs allows the determination of the PoA of Game 6 and its comparison with the PoA of Game 3. In order to derive such set of NE, first reformulate the cost function as follows:

$$
\phi\left(\underline{a}_{t}\right)=\phi\left(\underline{a}_{t-1}\right)+\psi\left(\underline{a}_{t}\right),
$$

with $\psi\left(\left(\underline{a}_{t}\right)= \begin{cases}\|\underline{M}(t)\|_{1}+\left\|\underline{a}_{t}\right\|_{1} & \text { if }\left\|\underline{a}_{t}\right\|_{1} \neq 1 \\ \left\|\underline{\mathcal{D}}\left(\underline{a_{t}}\right)\right\|_{1}+1 & \text { otherwise. }\end{cases}\right.$

Let $\underline{a}_{t}^{*}$ be an action profile such that $\left\|\underline{a}_{t}^{*}\right\|_{1}>2$. For any unilateral deviation of a transmitting player $i$, the cost function increases as follows:

$$
\begin{gathered}
\phi\left(\underline{a}_{t}^{*}\right)-\phi\left(\underline{a}_{t, i}, \underline{a}_{t,-i}^{*}\right)=\left\|\underline{a}_{t}^{*}\right\|_{1}-\left\|\left(\underline{a}_{t, i}, \underline{a}_{t,-i}^{*}\right)\right\|_{1}=1 \\
\forall a_{i}(t) \neq a_{i}^{*}(t) \in \mathcal{A}_{i}(t) \text { s.t. } a_{i}^{*}(t)=1 .
\end{gathered}
$$

The cost function increases by some unilateral deviation. Hence all action profile $\underline{a}_{t}^{*}$ such that $\left\|\underline{a}_{t}^{*}\right\|_{1}>2$ are no more NEs of the game. By the same argument used in Game 1, the action profiles $\underline{a}_{t}^{*}$ such that $\left\|\underline{a}_{t}^{*}\right\|_{1}=0$ or $\left\|\underline{a}_{t}^{*}\right\|_{1}=2$ are not NEs of the game.

For the action profile $\underline{a}_{t}^{*}$ such that $\left\|\underline{a}_{t}^{*}\right\|_{1}=1$, the difference of cost function for any unilateral deviation is the following:

$$
\begin{aligned}
& \phi\left(\underline{a}_{t, i}, \underline{a}_{t,-i}^{*}\right)-\phi\left(\underline{a}_{t}^{*}\right)= \\
& \left\{\begin{array}{c}
\|\underline{M}(t)\|_{1}-\left\|\underline{\mathcal{D}}\left(\underline{a_{t}}\right)\right\|_{1}+1>0 \\
\forall a_{i}(t) \neq a_{i}^{*}(t) \in \mathcal{A}_{i}(t), \quad \forall i \in \mathcal{M} \backslash\{j\} \\
\|\underline{M}(t)\|_{1}-\left\|\underline{\mathcal{D}}\left(\underline{a_{t}}\right)\right\|_{1}-1 \geq 0 \\
\forall a_{j}(t) \neq a_{j}^{*}(t) \in \mathcal{A}_{j}(t) .
\end{array}\right.
\end{aligned}
$$

Since no unilateral deviation can yield a lower cost, therefore all action profile $\underline{a}_{t}^{*}$ such that $\left\|\underline{a}_{t}^{*}\right\|_{1}=1$ are NEs of the game. The set of all NE of the Game 6 can be expressed as:

$$
\mathcal{E}^{S D D}(t)=\mathcal{O}(t)
$$

Substituting the above expression (F.4) in the PoA expression (25), the PoA of Game 6 can be expressed as follows:

$$
P o A_{6}(t)=\frac{\min _{\left\|\underline{t}_{t}\right\|_{1}=1} \phi\left(\underline{a}_{t-1}\right)+\left\|\underline{\mathcal{D}}\left(\underline{a}_{t}\right)\right\|_{1}+1}{\left\|\underline{\max }_{t}\right\|_{1=1} \phi\left(\underline{a}_{t-1}\right)+\left\|\underline{\mathcal{D}}\left(\underline{a_{t}}\right)\right\|_{1}+1} .
$$

Moreover, since $0 \leq\left\|\underline{\mathcal{D}}\left(\underline{a_{t}}\right)\right\|_{1} \leq M-1$ for $\left\|\underline{a}_{t}\right\|_{1}=$, the $P O A$ can be bounded by the following expressions:

$$
1-\frac{\|\underline{M}(t)\|_{1}-1}{\phi^{\prime}\left(\underline{a}_{t-1}\right)+\|\underline{M}(t)\|_{1}} \leq \operatorname{Po} A(t) \leq 1 .
$$

Using the expression of the PoA of Game 3 in (C.6), it can be quickly concluded that the PoA of Game 6 is more efficient than the one of Game 3. In other words, $0 \leq P_{o} A_{3}(t) \leq$ $P_{o} A_{6}(t) \leq 1$.

\section{REFERENCES}

[1] A. Douik, S. Sorour, H. Tembine, T. Y. Al-Naffouri, and M.-S. Alouini, "A game theoretic approach to minimize the completion time of network coded cooperative data exchange," in Proc. of IEEE Global Telecommunications Conference (GLOBECOM' 2014), Austin, Texas, USA, Dec. 2014.

[2] R. Ahlswede, N. Cai, S.-Y. Li, and R. Yeung, "Network information flow," IEEE Transactions on Information Theory, vol. 46, no. 4, pp. 1204-1216, Jul 2000.

[3] X. Li, C.-C. Wang, and X. Lin, "On the capacity of immediatelydecodable coding schemes for wireless stored-video broadcast with hard deadline constraints," IEEE Journal on Selected Areas in Communications, vol. 29, no. 5, pp. 1094-1105, May 2011.

[4] J.-S. Park, M. Gerla, D. Lun, Y. Yi, and M. Medard, "Codecast: a network-coding-based ad hoc multicast protocol," IEEE Wireless Communications, vol. 13, no. 5, pp. 76-81, October 2006.

[5] T. Ho, R. Koetter, M. Medard, D. Karger, and M. Effros, "The benefits of coding over routing in a randomized setting," in Proc. of IEEE International Symposium on Information Theory (ISIT' 2003), Yokohama, Japan, June 2003, pp. 442-448.

[6] S. Katti, H. Rahul, W. Hu, D. Katabi, M. Medard, and J. Crowcroft, "Xors in the air: Practical wireless network coding," IEEE/ACM Transactions on Networking, vol. 16, no. 3, pp. 497-510, June 2008.

[7] S. Katti, D. Katabi, W. Hu, H. Rahul, and M. Medard, "The Importance of Being Opportunistic: Practical Network Coding for Wireless Environments," in Proc. of Annual Allerton Conference on Communication, Control and Computing (Allerton' 2005), Monticello, Illinois, USA, 2005.

[8] S. Sorour and S. Valaee, "Minimum broadcast decoding delay for generalized instantly decodable network coding," in Proc. of IEEE Global Telecommunications Conference (GLOBECOM' 2010), Miami, Florida, USA, Dec 2010, pp. 1-5. 
[9] A. Douik, S. Sorour, T. Al-Naffouri, and M.-S. Alouini, "A lossy graph model for delay reduction in generalized instantly decodable network coding," IEEE Wireless Communications Letters, vol. 3, no. 3, pp. 281284, June 2014

[10] P. Sadeghi, R. Shams, and D. Traskov, "An optimal adaptive network coding scheme for minimizing decoding delay in broadcast erasure channels," EURASIP Journal on Wireless Communications and Networking, vol. 2010, pp. 1-14, 2010.

[11] L. Lu, M. Xiao, and L. K. Rasmussen, "Design and analysis of relayaided broadcast using binary network codes," Journal of Communications, vol. 6, no. 8, pp. 610-617, 2011.

[12] N. Aboutorab, P. Sadeghi, and S. Tajbakhsh, "Instantly decodable network coding for delay reduction in cooperative data exchange systems," in Proc. of IEEE International Symposium on Information Theory (ISIT' 2013), Istanbul, Turkey, July 2013, pp. 3095-3099.

[13] M. Muhammad, M. Berioli, G. Liva, and G. Giambene, "Instantly decodable network coding protocols with unequal error protection," in Proc. of IEEE International Conference on Communications (ICC' 2013), Sydney, Australia, June 2013, pp. 5120-5125.

[14] A. Douik, S. Sorour, T. Y. Al-Naffouri, and M.-S. Alouini, "Instantly decodable network coding for real-time device-to-device communications," EURASIP Journal on Advances in Signal Processing, vol. 2016, no. 1, pp. 1-14, 2016.

[15] Y. Liu and C. W. Sung, "Quality-aware instantly decodable network coding," IEEE Transactions on Wireless Communications, vol. 13, no. 3, pp. 1604-1615, March 2014.

[16] A. Douik, S. Sorour, T. Y. Al-Naffouri, H.-C. Yang, and M.-S. Alouini, "Delay reduction in Multi-Hop Device-to-Device communication using network coding," in Proc. of IEEE International Symposium on Network Coding (NetCod' 2015), Sydney, Australia.

[17] A. Douik, S. Sorour, M.-S. Alouini, and T. Al-Naffouri, "Delay reduction in lossy intermittent feedback for generalized instantly decodable network coding," in Proc. of IEEE 9th International Conference on Wireless and Mobile Computing, Networking and Communications (WiMob' 2013), Lyon, France, Oct 2013, pp. 388-393.

[18] M. Esmaeilzadeh and P. Sadeghi, "Optimizing completion delay in network coded systems over TDD erasure channels with memory," in Proc. of IEEE International Symposium on Communications and Information Technologies (ISCIT' 2012), Queensland, Australia, Oct 2012, pp. 883-888.

[19] A. Douik, S. Sorour, M. Alouini, and T. Y. Al-Naffouri, "On minimizing the maximum broadcast decoding delay for instantly decodable network coding," Proc. of IEEE Vehicular Technology Conference (VTC-Fall' 2014), Vancouver, BC, Canada, vol. abs/1404.0265, 2014.

[20] A. Douik, S. Sorour, M.-S. Alouini, and T. Y. Al-Naffouri, "Completion time reduction in instantly decodable network coding through decoding delay control," in Proc. of IEEE Global Telecommunications Conference (GLOBECOM' 2014), Austin, Texas, USA, Dec. 2014.

[21] S. Sorour and S. Valaee, "On minimizing broadcast completion delay for instantly decodable network coding," in Proc. of IEEE International Conference on Communications (ICC'2010), Cape Town, South Africa, May 2010, pp. 1-5.

[22] S. Sorour, A. Douik, S. Valaee, T. Al-Naffouri, and M. Alouini, "Partially blind instantly decodable network codes for lossy feedback environment," IEEE Transactions on Wireless Communications, vol. 13, no. 9, pp. 4871-4883, Sept 2014.

[23] J. Andrews, S. Buzzi, W. Choi, S. Hanly, A. Lozano, A. Soong, and J. Zhang, "What will 5G be?" IEEE Journal on Selected Areas in Communications, vol. 32, no. 6, pp. 1065-1082, June 2014.

[24] S. Tajbakhsh and P. Sadeghi, "Coded cooperative data exchange for multiple unicasts," in Proc. of IEEE Information Theory Workshop (ITW' 2012), Lausanne, Switzerland, Sept 2012, pp. 587-591.

[25] A. Douik, S. Sorour, T. Y. Al-Naffouri, and M.-S. Alouini, "Delay reduction for instantly decodable network coding in persistent channels with feedback imperfections," IEEE Transactions on Wireless Communications, Vol. 14, No. 11, November 2015.

[26] C. Fragouli, D. Lun, M. Medard, and P. Pakzad, "On feedback for network coding," in Proc. of 41st Annual Conference on Information Sciences and Systems (CISS' 2007), Hodson, California, USA, March 2007, pp. 248-252.

[27] L. Keller, E. Drinea, and C. Fragouli, "Online broadcasting with network coding," in IEEE 4th Workshop on Network Coding, Theory and Applications (NetCod' 2008), Hong Kong, China, Jan 2008, pp. 1-6.

[28] R. Costa, D. Munaretto, J. Widmer, and J. Barros, "Informed network coding for minimum decoding delay," in Proc. of the 5th IEEE International Conference on Mobile Ad Hoc and Sensor Systems (MASS' 2008), Atlanta, Georgia, USA, Sept 2008, pp. 80-91.
[29] S. Lasaulce and H. Tembine, Game Theory and Learning for Wireless Networks: Fundamentals and Applications, 1st ed. Academic Press, 2011.

[30] D. Monderer, "Potential games," Games and Economic Behavior, vol. 14, no. 1, pp. $124-143,1996$.

[31] G. Scutari, S. Barbarossa, and D. Palomar, "Potential games: A framework for vector power control problems with coupled constraints," in Proc. of IEEE International Conference on Acoustics, Speech and Signal Processing (ICASSP' 2006), Toulouse, France, vol. 4, May 2006, pp. IV-IV.

[32] E. Koutsoupias and C. Papadimitriou, "Worst-case equilibria," in Proc. of the 16th Annual Symposium On Theoretical Aspects Of Computer Science (STACS' 1999), Trier, Germany, 1999, pp. 404-413.

[33] J. W. Friedman, "The legacy of Augustin Cournot," Cahiers d'conomie Politique, vol. 37, no. 1, pp. 31-46, 2000.

[34] H. Tembine, Distributed Strategic Learning for Wireless Engineers. CRC Press, Inc., 2012.

[35] L. P. Kaelbling, M. L. Littman, and A. W. Moore, "Reinforcement learning: a survey," Journal of Artificial Intelligence Research, vol. 4, pp. 237-285, 1996.

[36] R. Bush and F. Mosteller., Stochastic models of learning. Wiley Sons, New York, 1955.

[37] A. Douik, S. Sorour, T. Y. Al-Naffouri, and M.-S. Alouini, "Decoding delay controlled completion time reduction in instantly decodable network coding," IEEE Transactions on Vehicular Technology, 2016.

[38] T. Borgers, "Learning through reinforcement and replicator dynamics," Journal of Economic Theory, vol. 77, no. 1, pp. 1 - 14, 1997.

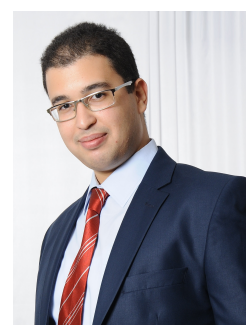

Ahmed Douik (S'13) received the Eng. degree in electronic and communication engineering (with first class honors) from the Ecole Polytechnique de Tunisie, Tunisia, in 2013, the M.S. degree in electrical engineering from King Abdullah University of Science and Technology, Thuwal, Saudi Arabia, in 2015. He is now pursuing his Ph.D. at the California Institute of Technology, Pasadena, CA, USA. His research interests include cloud-radio access networks, network coding, single and multi-hop transmissions, and cooperation communication.

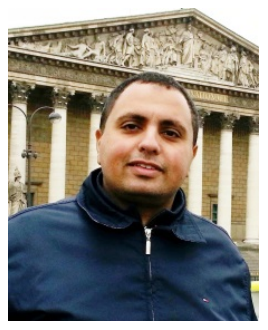

Sameh Sorour (S98-M11-SM16) is an Assistant Professor at the Department of Electrical and Computer Engineering, University of Idaho. He received his B.Sc. and M.Sc. degrees in Electrical Engineering from Alexandria University, Egypt, in 2002 and 2006, respectively. In 2011, he obtained his Ph.D. degree in Electrical and Computer Engineering from University of Toronto, Canada. After two postdoctoral fellowships at University of Toronto and King Abduallah University of Science and Technology (KAUST), he joined King Fahd University of Petroleum and Minerals (KFUPM) in 2013 before moving to University of Idaho in 2016. His research interests include network coding, data dissemination and exchange for the Internet of Things, cloud storage networks, femtocaching, smart management of networks and cities, and indoor positioning. 


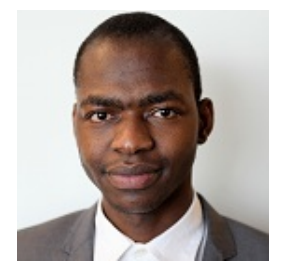

Hamidou Tembine (S06-M10-SM13) received the M.S. degree from Ecole Polytechnique, Paris, France, and the Ph.D. degree from the University of Avignon, Avignon, France. His current research interests include evolutionary games, mean field stochastic games and distributed strategic learning and applications. He has co-authored two books.

Mr. Tembine received the Outstanding Young Researcher Award from IEEE ComSoc in 2014. He was the recipient of five Best Paper Awards.

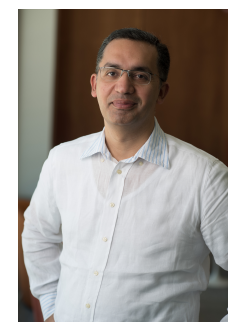

Tareq Y. Al-Naffouri (M'10) Tareq Al-Naffouri received the B.S. degrees in mathematics and electrical engineering (with first honors) from King Fahd University of Petroleum and Minerals, Dhahran, Saudi Arabia, the M.S. degree in electrical engineering from the Georgia Institute of Technology, Atlanta, in 1998, and the Ph.D. degree in electrical engineering from Stanford University, Stanford, CA, in 2004.

He was a visiting scholar at California Institute of Technology, Pasadena, CA, from January to August 2005 and during summer 2006. He was a Fulbright scholar at the University of Southern California from February to September 2008. He has held internship positions at NEC Research Labs, Tokyo, Japan, in 1998, Adaptive Systems Lab, University of California at Los Angeles in 1999, National Semiconductor, Santa Clara, CA, in 2001 and 2002, and Beceem Communications Santa Clara, CA, in 2004. He is currently an Associate at the Electrical Engineering Department, King Abdullah University of Science and Technology (KAUST). His research interests lie in the areas of sparse, adaptive, and statistical signal processing and their applications and in network information theory. He has over 150 publications in journal and conference proceedings, 9 standard contributions, 10 issued patents, and 6 pending.

Dr. Al-Naffouri is the recipient of the IEEE Education Society Chapter Achievement Award in 2008 and Al-Marai Award for innovative research in communication in 2009. Dr. Al-Naffouri has also been serving as an Associate Editor of Transactions on Signal Processing since August 2013.

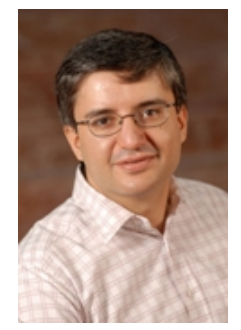

Mohamed-Slim Alouini (S'94-M'98-SM'03-F'09) was born in Tunis, Tunisia. He received the Ph.D. degree in electrical engineering from the California Institute of Technology (Caltech), Pasadena, CA, USA, in 1998. He served as a faculty member at the University of Minnesota, Minneapolis, MN, USA, then in the Texas A\&M University at Qatar, Education City, Doha, Qatar, before joining King Abdullah University of Science and Technology (KAUST), Thuwal, Makkah Province, Saudi Arabia, as a Professor of electrical engineering in 2009. His current research interests include the modeling, design, and performance analysis of wireless communication systems. 